\title{
Free Vibration and Damping of Rotating Composite Shaft with a Constrained Layer Damping
}

\author{
Yongsheng Ren and Yuhuan Zhang \\ College of Mechanical and Electronic Engineering, Shandong University of Science and Technology, Qingdao 266590, China \\ Correspondence should be addressed to Yongsheng Ren; rys56@sohu.com
}

Received 13 November 2015; Revised 28 January 2016; Accepted 8 February 2016

Academic Editor: Sergio De Rosa

Copyright ( 2016 Y. Ren and Y. Zhang. This is an open access article distributed under the Creative Commons Attribution License, which permits unrestricted use, distribution, and reproduction in any medium, provided the original work is properly cited.

\begin{abstract}
The free vibration and damping characteristics of rotating shaft with passive constrained layer damping (CLD) are studied. The shaft is made of fiber reinforced composite materials. A composite beam theory taking into account transverse shear deformation is employed to model the composite shaft and constraining layer. The equations of motion of composite rotating shaft with CLD are derived by using Hamilton's principle. The general Galerkin method is applied to obtain the approximate solution of the rotating CLD composite shaft. Numerical results for the rotating CLD composite shaft with simply supported boundary condition are presented; the effects of thickness of constraining layer and viscoelastic damping layers, lamination angle, and rotating speed on the natural frequencies and modal dampings are discussed.
\end{abstract}

\section{Introduction}

Rotating shafts are used for power transmission in helicopter drive applications. Weight, vibration, strength, and fatigue have been recognized as serious problem in structural design of this device. Use of fiber reinforced composite materials has received great interest in helicopter drive shaft because composite materials have low weight, high stiffness-to-weight ratio, and vibration damping characteristics compared to metals.

Dynamics of rotating composite material shaft have been an important issue in the field of rotor dynamics. Zinberg and Symonds [1] used an equivalent modulus beam theory (EMBT) to predict the critical speeds of composite shaft. dos Reis et al. [2] analyzed the critical speeds of thinwalled composite shafts by incorporating the Timoshenko beam theory with the Donnell thin shell theory. Chang et al. [3] presented a simple rotating composite shaft model. The composite shaft has rigid disks and is supported in linear elastic bearings. The free vibration of a rotating tapered composite Timoshenko shaft was investigated by Kim and his coworkers [4]. The effects of shaft tapering on the bending natural frequencies have been examined. Singh and
Gupta [5] used equivalent modulus beam theory and a layerwise beam theory (LBT) for the rotor dynamics analysis of a composite shaft supported on general eight coefficient bearings. Gubran and Gupta [6] presented EMBT. The effect of stacking sequence and coupling mechanisms on the natural frequencies of composite shafts was studied. Song et al. [7] proposed a rotating composite shaft model using a thinwalled composite beam theory for the vibration and stability analysis. Sino et al. [8] studied the dynamic instability of rotating composite shaft using a homogenized finite element beam model, which takes into account internal damping. Ren et al. [9] presented a structural modelling and dynamical analysis of rotating composite Timoshenko shaft based on the thin-walled composite beam theory referred to as variational asymptotic method.

In order to suppress large amplitudes of the shaft of passage through resonance, an effective method is required. CLD treatment has been widely used for damping out structural vibration. Its practical application can be found in aerospace, vehicles, submarines, and so forth. The structures with CLD treatment consist of base structure, viscoelastic material (VEM) layer, and constraining layer (CL). The VEM layer is sandwiched between base structure and CL. The VEM 


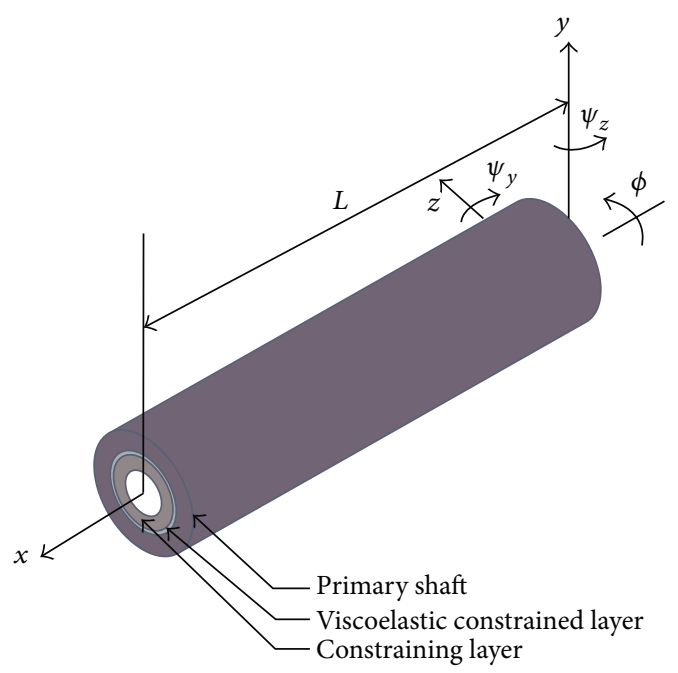

(a)

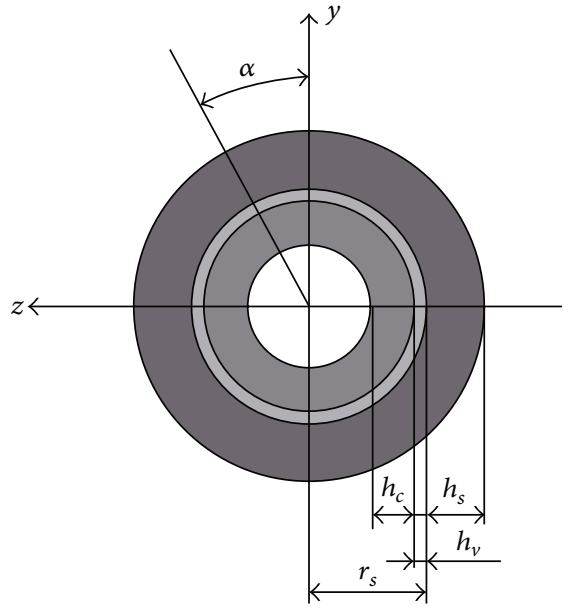

(b)

FIGURE 1: Geometric configuration of the composite shaft with a constrained damping layer.

undergoes shear strain as the base structure bends by which the vibration energy of the base structure can be dissipated.

The dynamics of beam, plate, and shell structures with CLD have been studied by many authors. Rao [10] presented an analytical model to investigate the frequencies and loss factors of CLD beam under various boundary conditions. Bhimaraddi [11] calculated both natural frequencies and loss factor CLD sandwich beam with simply supported boundary condition. Sakiyama et al. [12] used the discrete green function for the free vibration analysis of sandwich beams with elastic or viscoelastic VEM core. Fasana and Marchesiello [13] used Rayleigh-Ritz method to study the free vibration of sandwich CLD beams. Ramesh and Ganesan [14] evaluated the vibrational response of CLD cylindrical shells to different types of loading. Wang and Chen [15] presented a finite element model of a cylindrical shell with partially CLD by using a discrete layer theory. Garg et al. [16] studied the free vibration of simply supported composite and sandwich doubly curved shell based on Sander's theory. Gao and Shen [17] presented the natural frequencies and loss factors of nonsymmetric composite plates with active CLD. Chen and Huang [18] used the energy approach for vibration analysis of plates with partially active CLD. Liu et al. [19] developed a dynamical finite element model of rotating cantilever plates with active CLD. Cao et al. [20] reported a parametric study for free vibration characteristics of a cylindrical shell with CLD. Jin et al. [21] developed an accurate solution for the vibration and damping of sandwich cylindrical shells with arbitrary boundary conditions.

Studies on the shaft with CLD, especially the rotating composite shaft with CLD, were relatively limited. Napolitano et al. [22] presented torsion damping analysis for the composite shaft with the extension-twist coupling and embedded CLD, respectively. The finite element and experimental results showed that significant torsion damping can be achieved by both design concepts. Venkatachalam and Prabu [23] conducted experimental and numerical study for the composite shaft-disk system with various CLD, including VEM, electrorheological and magnetorheological fluid. The semianalytical finite element method based on shell theory has been employed to solve natural frequencies and loss factors. Ghoneim and Lawrie [24] derived the transverse bending vibration of partially CLD composite shaft by the dynamic equilibrium and Timoshenko beam assumption. Applying the finite element method and the assumed mode method, the damping capability of the shaft was illustrated.

In the present work, an analytical model is developed for free vibration and damping capacity analysis of a rotating CLD composite shaft. The base composite shaft and constraining layer are modelled by using a first-order beam theory in conjunction with the constitutive equations of laminated composite based on three-dimensional continua and the strain-displacement relations. The equations of motion of the rotating CLD composite shaft with extensiontwist-bending coupling are derived by employing Hamilton's principle. The approximate solutions of the shaft system are obtained using the general Galerkin method. Numerical results for the rotating CLD composite shaft with simply supported boundary condition are presented, and the effects of thickness of constraining layer and viscoelastic damping layers, lamination angle, and rotation on the natural frequencies and modal dampings are discussed.

\section{Theoretical Formulations}

2.1. Basic Assumptions. The following assumptions will be adopted for the structural modeling of the constrained layer damping shaft as shown in Figure 1:

(a) The base composite shaft and constraining layer satisfy Timoshenko beam assumption.

(b) The shaft, constraining layer, and viscoelastic damping layer have the same transverse displacements $u_{y}$ and $u_{z}$. 
(c) Only shear strains are considered for the viscoelastic damping layer.

2.2. Constitutive Relation. The constitutive relation for a lamina with respect to the material coordinated system $(1,2,3)$ is

$$
\begin{aligned}
& {\left[\begin{array}{llllll}
\sigma_{11} & \sigma_{22} & \sigma_{33} & \tau_{23} & \tau_{31} & \tau_{12}
\end{array}\right]^{T}} \\
& \quad=\left[\begin{array}{llllll}
Q_{i j}
\end{array}\right]\left[\begin{array}{llllll}
\varepsilon_{11} & \varepsilon_{22} & \varepsilon_{33} & \gamma_{23} & \gamma_{31} & \gamma_{12}
\end{array}\right]^{T},
\end{aligned}
$$

where $\left[Q_{i j}\right]$ is the stiffness matrix.

The constitutive relations for an arbitrary lamina in the base composite shaft and the constraining layer with respect to the cylindrical coordinated system $(x, r, \alpha)$ can be written as

$$
\begin{aligned}
& {\left[\begin{array}{llllll}
\sigma_{r} & \sigma_{\alpha} & \sigma_{x} & \tau_{\alpha x} & \tau_{x r} & \tau_{r \alpha}
\end{array}\right]^{T}} \\
& =\left[\bar{Q}_{i j}\right]\left[\begin{array}{llllll}
\varepsilon_{r} & \varepsilon_{\alpha} & \varepsilon_{x} & \gamma_{\alpha x} & \gamma_{x r} & \gamma_{r \alpha}
\end{array}\right]^{T},
\end{aligned}
$$

where $\left[\bar{Q}_{i j}\right]=\left[T_{\theta}\right]^{-1}\left[Q_{i j}\right]\left[T_{\theta}\right]$ is the transformed stiffness of a lamina and $\theta$ the fiber angle between the $x$-axis and the fiber direction.

In beam theories, $\sigma_{r}, \sigma_{\alpha}$, and $\tau_{r \alpha}$ are taken to be zero. However, considering the stress $\sigma_{r}, \sigma_{\alpha}$ cannot be zero due to the high speed rotating's effect and so we will retain them in the current work. The specific forms of $\sigma_{r}$ and $\sigma_{\alpha}$ are left unspecified and the only assumption made is that they are axisymmetric. From the first, second, and sixth equations of (2), $\varepsilon_{r}, \varepsilon_{\alpha}$, and $\gamma_{r \alpha}$ can be expressed in terms of $\varepsilon_{x}, \gamma_{\alpha x}, \gamma_{x r}, \sigma_{r}$, and $\sigma_{\alpha}$. By substituting the resulting expressions into the third, fourth, and fifth equations of (2) and dropping $\varepsilon_{r}, \varepsilon_{\alpha}$, and $\gamma_{r \alpha}$, one has

$$
\left[\begin{array}{c}
\sigma_{x} \\
\tau_{\alpha x} \\
\tau_{x r}
\end{array}\right]=\left[\begin{array}{lll}
\bar{k}_{11} & \bar{k}_{12} & \bar{k}_{13} \\
\bar{k}_{21} & \bar{k}_{22} & \bar{k}_{23} \\
\bar{k}_{31} & \bar{k}_{32} & \bar{k}_{33}
\end{array}\right]\left[\begin{array}{l}
\varepsilon_{x} \\
\gamma_{\alpha x} \\
\gamma_{x r}
\end{array}\right]+\left[\begin{array}{ll}
\bar{c}_{11} & \bar{c}_{12} \\
\bar{c}_{21} & \bar{c}_{22} \\
\bar{c}_{31} & \bar{c}_{32}
\end{array}\right]\left[\begin{array}{l}
\sigma_{r} \\
\sigma_{\alpha}
\end{array}\right],
$$

where $\bar{k}_{i j}$ and $\bar{c}_{i j}$ are known stiffness coefficients and are given in Appendix B. Employing the Timoshenko beam theory in the base composite shaft and the constraining layer, the strain components in (3) can be expressed as

$$
\begin{aligned}
\varepsilon_{x}= & \frac{\partial u_{x}}{\partial x}-\frac{\partial \psi_{y}}{\partial x} r \cos \alpha+\frac{\partial \psi_{z}}{\partial x} r \sin \alpha, \\
\gamma_{x r}= & \left(\frac{\partial u_{y}}{\partial x}-\psi_{y}\right) \cos \alpha+\left(\frac{\partial u_{z}}{\partial x}+\psi_{z}\right) \sin \alpha, \\
\gamma_{\alpha x}= & -\left(\frac{\partial u_{y}}{\partial x}-\psi_{y}\right) \sin \alpha+\left(\frac{\partial u_{z}}{\partial x}+\psi_{z}\right) \cos \alpha \\
& +r \frac{\partial \phi}{\partial x},
\end{aligned}
$$

where $u_{x}, u_{y}$, and $u_{z}$ denote the displacements of the neutral axis in $x, y$, and $z$ directions, respectively, $\psi_{y}$ and $\psi_{z}$ denote the rotation angles about the $z$ - and $y$-axis, respectively, and $\phi$ is the twist angle about $x$-axis.

The Timoshenko shear correction factor $\kappa$ can be incorporated into constitutive equations (3) as [4]

$$
\begin{aligned}
{\left[\begin{array}{c}
\sigma_{x} \\
\tau_{\theta x} \\
\tau_{x r}
\end{array}\right]=} & {\left[\begin{array}{lll}
\bar{k}_{11} & \kappa \bar{k}_{12} & \kappa \bar{k}_{13} \\
\bar{k}_{21} & \kappa \bar{k}_{22} & \kappa \bar{k}_{23} \\
\bar{k}_{31} & \kappa \bar{k}_{32} & \kappa \bar{k}_{33}
\end{array}\right]\left[\begin{array}{c}
\varepsilon_{x} \\
\gamma_{\theta x}^{B} \\
\gamma_{x r}^{B}
\end{array}\right] } \\
& +\left[\begin{array}{lll}
0 & \bar{k}_{12} & 0 \\
0 & \bar{k}_{22} & 0 \\
0 & \bar{k}_{32} & 0
\end{array}\right]\left[\begin{array}{c}
0 \\
\gamma_{\theta x}^{T} \\
0
\end{array}\right]+\left[\begin{array}{ll}
\bar{c}_{11} & \bar{c}_{12} \\
\bar{c}_{21} & \bar{c}_{22} \\
\bar{c}_{31} & \bar{c}_{32}
\end{array}\right]\left[\begin{array}{l}
\sigma_{r} \\
\sigma_{\theta}
\end{array}\right],
\end{aligned}
$$

where $\gamma_{x r}^{B}=\gamma_{x r}$ and $\gamma_{\alpha x}^{B}=\gamma_{\alpha x}-r \phi^{\prime}$ and the superscripts $B$ and $T$ denote bending and torsional contributions, respectively.

The constitutive relations in the viscoelastic layer are

$$
\begin{aligned}
& \tau_{x \alpha}^{v}=G_{v} \gamma_{x \alpha}^{v}, \\
& \tau_{r x}^{v}=G_{v} \gamma_{r x}^{v},
\end{aligned}
$$

where $G_{v}$ is the complex shear modulus of the viscoelastic layer.

The strain-displacement relations of the viscoelastic layer can be written as

$$
\begin{aligned}
\gamma_{r x}^{v}= & {\left[u_{z}^{\prime}+\frac{\left(r_{s} \psi_{z}^{s}-r_{c} \psi_{z}^{c}\right)}{h_{v}}\right] \sin \alpha } \\
& +\left[u_{y}^{\prime}-\frac{\left(r_{s} \psi_{y}^{s}-r_{c} \psi_{y}^{c}\right)}{h_{v}}\right] \cos \alpha, \\
\gamma_{x \alpha}^{v}= & {\left[u_{z}^{\prime}+\frac{\left(\psi_{z}^{s}+\psi_{z}^{c}\right)}{2}\right] \cos \alpha } \\
& -\left[u_{y}^{\prime}-\frac{\left(\psi_{y}^{s}+\psi_{y}^{c}\right)}{2}\right] \sin \alpha,
\end{aligned}
$$

where the superscripts or subscripts $s, c$, and $v$ denote the shaft, constraining layer, and viscoelastic layer, respectively, and $r_{s}$ and $r_{c}$ are the radius of the inner surface of the constraining layer and the outer surface of the shaft.

2.3. Kinetic Energy and Strain Energy. Kinetic energy of the base composite shaft, constraining layer, and viscoelastic layer is 


$$
\begin{aligned}
T_{s}= & \frac{1}{2} \\
& \cdot \int_{0}^{L} \int_{0}^{2 \pi} \sum_{k=1}^{n} \int_{r_{k-1}}^{r_{k}} \rho_{s}^{(k)}\left\{\left[\dot{u}_{x}^{s}+r\left(\dot{\psi}_{z}^{s}+\Omega \psi_{y}^{s}\right) \sin \alpha-r\left(\dot{\psi}_{y}^{s}-\Omega \psi_{z}^{s}\right) \cos \alpha\right]^{2}+\left(\dot{u}_{y}-r \dot{\alpha} \sin \alpha\right)^{2}+\left(\dot{u}_{z}+r \dot{\alpha} \cos \alpha\right)^{2}\right\} r d r d \alpha d x \\
= & \frac{1}{2} \int_{0}^{L}\left\{m_{s}\left(\dot{u}_{x}^{s 2}+\dot{u}_{y}^{2}+\dot{u}_{z}^{2}\right)+I_{s}\left[2\left(\Omega+\dot{\phi}_{s}\right)^{2}+\dot{\psi}_{y}^{s 2}+\dot{\psi}_{z}^{s 2}+2 \Omega \dot{\psi}_{z}^{s} \psi_{y}^{s}-2 \Omega \dot{\psi}_{y}^{s} \psi_{z}^{s}\right]\right\} d x \\
T_{c}= & \frac{1}{2} \\
& \cdot \int_{0}^{L} \int_{0}^{2 \pi} \sum_{k=1}^{n} \int_{r_{k-1}}^{r_{k}} \rho_{c}^{(k)}\left\{\left[\dot{u}_{x}^{c}+r\left(\dot{\psi}_{z}^{c}+\Omega \psi_{y}^{c}\right) \sin \alpha-r\left(\dot{\psi}_{y}^{c}-\Omega \psi_{z}^{c}\right) \cos \alpha\right]^{2}+\left(\dot{u}_{y}-r \dot{\alpha} \sin \alpha\right)^{2}+\left(\dot{u}_{z}+r \dot{\alpha} \cos \alpha\right)^{2}\right\} r d r d \alpha d x \\
= & \frac{1}{2} \int_{0}^{L}\left\{m_{c}\left(\dot{u}_{x}^{c 2}+\dot{u}_{y}^{2}+\dot{u}_{z}^{2}\right)+I_{c}\left[2\left(\Omega+\dot{\phi}_{c}\right)^{2}+\dot{\psi}_{y}^{c 2}+\dot{\psi}_{z}^{c 2}+2 \Omega \dot{\psi}_{z}^{c} \psi_{y}^{c}-2 \Omega \dot{\psi}_{y}^{c} \psi_{z}^{c}\right]\right\} d x \\
T_{v}= & \frac{1}{2} \int_{0}^{L}\left[m_{v}\left(\dot{u}_{x}^{v 2}+\dot{u}_{y}^{2}+\dot{u}_{z}^{2}\right)+2 \Omega I_{v}\left(\Omega+\dot{\phi}_{v}\right)^{2}\right] d x,
\end{aligned}
$$

where $m_{l}=\pi \sum_{k=1}^{n} \rho_{l}^{(k)}\left(r_{k}^{2}-r_{k-1}^{2}\right)$ and $I_{l}=(\pi / 4) \sum_{k=1}^{n} \rho_{l}^{(k)}\left(r_{k}^{4}-\right.$ $\left.r_{k-1}^{4}\right)(l=s, c)$ are the mass and the polar mass of inertia per unit length, respectively, and $\rho_{l}^{(k)}$ is the mass density of the $k$ th layer. $m_{v}=\rho_{v} \pi\left(r_{s}^{2}-r_{c}^{2}\right)=\rho_{v} A_{v}$ and $I_{l}=(\pi / 4) \rho_{v}\left(r_{s}^{4}-\right.$ $r_{c}^{4}$ ) denote the mass and the polar mass of inertia per unit length of the viscoelastic layer, respectively. For simplicity, the approximate expressions $\dot{u}_{x}^{v}=\left(\dot{u}_{x}^{s}+\dot{u}_{x}^{c}\right) / 2$ and $\dot{\phi}_{v}=\left(\dot{\phi}_{v}^{s}+\dot{\phi}_{v}^{c}\right) / 2$ will be used in the further analysis.

Strain energy of the base composite shaft, constraining layer, and viscoelastic layer is

$$
U_{s}=\frac{1}{2} \int_{0}^{L} \int_{0}^{2 \pi} \sum_{k=1}^{n} \int_{r_{k-1}}^{r_{k}}\left(\sigma_{x}^{s} \varepsilon_{x}^{s}+\tau_{r x}^{s} \gamma_{r x}^{s}+\tau_{x \alpha}^{s} \gamma_{x \alpha}^{s}\right)
$$

- $r d r d \alpha d x$

$$
U_{c}=\frac{1}{2} \int_{0}^{L} \int_{0}^{2 \pi} \sum_{k=1}^{n} \int_{r_{k-1}}^{r_{k}}\left(\sigma_{x}^{c} \varepsilon_{x}^{c}+\tau_{r x}^{c} \gamma_{r x}^{c}+\tau_{x \alpha}^{c} \gamma_{x \alpha}^{c}\right)
$$

$\cdot r d r d \alpha d x$

$$
U_{v}=\frac{1}{2} \int_{0}^{L} \int_{0}^{2 \pi} \sum_{k=1}^{n} \int_{r_{k-1}}^{r_{k}}\left(\tau_{r x}^{v} \gamma_{r x}^{v}+\tau_{x \alpha}^{v} \gamma_{x \alpha}^{v}\right) r d r d \alpha d x .
$$

2.4. Motion Equations and Boundary Conditions. Taking the variation of (9) and (10) and considering (4), one has

$$
\begin{aligned}
\delta U_{l} & =\int_{0}^{L} \int_{0}^{2 \pi} \sum_{k=1}^{n} \int_{r_{k-1}}^{r_{k}}\left(\sigma_{x}^{l} \delta \varepsilon_{x}^{l}+\tau_{r x}^{l} \delta \gamma_{r x}^{l}+\tau_{x \alpha}^{l} \delta \gamma_{x \alpha}^{l}\right) r d r d \alpha d x \\
& =\int_{0}^{L} \int_{0}^{2 \pi} \sum_{k=1}^{n} \int_{r_{k-1}}^{r_{k}}\left\{\sigma_{x}^{l}\left[\frac{\partial \delta u_{x}^{l}}{\partial x}-\frac{\partial \delta \psi_{y}^{l}}{\partial x} r \cos \alpha+\frac{\partial \delta \psi_{z}^{l}}{\partial x} r \sin \alpha\right]\right.
\end{aligned}
$$

$$
\begin{aligned}
& +\tau_{r x}^{l}\left[\left(\frac{\partial \delta u_{y}}{\partial x}-\delta \psi_{y}^{l}\right) \cos \alpha+\left(\frac{\partial \delta u_{z}}{\partial x}+\delta \psi_{z}^{l}\right) \sin \alpha\right] \\
& +\tau_{x \alpha}^{l}\left[-\left(\frac{\partial \delta u_{y}}{\partial x}-\delta \psi_{y}^{l}\right) \sin \alpha+\left(\frac{\partial \delta u_{z}}{\partial x}+\delta \psi_{z}^{l}\right) \cos \alpha\right. \\
& \left.\left.+r \frac{\partial \delta \phi_{l}}{\partial x}\right]\right\} r d r d \alpha d x \quad(l=s, c) .
\end{aligned}
$$

Define the stress resultants $N_{x}^{l}, V_{y}^{l}$, and $V_{z}^{l}$ and stress couples $M_{x}^{l}, M_{y}^{l}$, and $M_{z}^{l}$ as follows:

$$
\begin{aligned}
& N_{x}^{l}=\int_{0}^{2 \pi} \sum_{k=1}^{n} \int_{r_{k-1}}^{r_{k}} \sigma_{x}^{l} r d r d \alpha, \\
& V_{y}^{l}=\int_{0}^{2 \pi} \sum_{k=1}^{n} \int_{r_{k-1}}^{r_{k}}\left(\tau_{r x}^{l} \cos \alpha-\tau_{x \alpha}^{l} \sin \alpha\right) r d r d \alpha, \\
& V_{z}^{l}=\int_{0}^{2 \pi} \sum_{k=1}^{n} \int_{r_{k-1}}^{r_{k}}\left(\tau_{r x}^{l} \sin \alpha+\tau_{x \alpha}^{l} \cos \alpha\right) r d r d \alpha, \\
& M_{x}^{l}=\int_{0}^{2 \pi} \sum_{k=1}^{n} \int_{r_{k-1}}^{r_{k}} \tau_{x \alpha}^{l} r^{2} d r d \alpha, \\
& M_{y}^{l}=\int_{0}^{2 \pi} \sum_{k=1}^{n} \int_{r_{k-1}}^{r_{k}} \sigma_{x}^{l} r^{2} \sin \alpha d r d \alpha, \\
& M_{z}^{l}=\int_{0}^{2 \pi} \sum_{k=1}^{n} \int_{r_{k-1}}^{r_{k}} \sigma_{x}^{l} r^{2} \cos \alpha d r d \alpha,
\end{aligned}
$$


Using the expressions given in (13), (12) can be written as

$$
\begin{aligned}
& \delta U_{l}=\int_{0}^{L}\left(N_{x}^{l} \frac{\partial \delta u_{x}^{l}}{\partial x}-M_{z}^{l} \frac{\partial \delta \psi_{y}^{l}}{\partial x}+M_{y}^{l} \frac{\partial \delta \psi_{z}^{l}}{\partial x}\right. \\
& +V_{y}^{l} \frac{\partial \delta u_{y}}{\partial x}+V_{z}^{l} \frac{\partial \delta u_{z}}{\partial x}-V_{y}^{l} \delta \psi_{y}^{l}+V_{z}^{l} \delta \psi_{z}^{l} \\
& \left.+M_{x}^{l} \frac{\partial \delta \phi_{l}}{\partial x}\right) d x, \quad(l=s, c) .
\end{aligned}
$$

Substituting (5) into (13), the stress resultants and stress couples can be obtained as

$$
\begin{aligned}
N_{x}^{l}= & 2 A_{11}^{l} \frac{\partial u_{x}^{l}}{\partial x}+2 B_{12}^{l} \frac{\partial \phi_{l}}{\partial x}+K_{N \Omega}^{l} \\
V_{y}^{l}= & -B_{31}^{l} \frac{\partial \psi_{y}^{l}}{\partial x}-B_{21}^{l} \frac{\partial \psi_{z}^{l}}{\partial x} \\
& +\kappa\left(A_{22}^{l}+A_{33}^{l}\right)\left(\frac{\partial u_{y}}{\partial x}-\psi_{y}^{l}\right) \\
& +\kappa\left(A_{32}^{l}-A_{23}^{l}\right)\left(\frac{\partial u_{z}}{\partial x}+\psi_{z}^{l}\right) \\
V_{z}^{l}= & B_{31}^{l} \frac{\partial \psi_{z}^{l}}{\partial x}-B_{21}^{l} \frac{\partial \psi_{y}^{l}}{\partial x} \\
& +\kappa\left(A_{22}^{l}+A_{33}^{l}\right)\left(\frac{\partial u_{z}}{\partial x}+\psi_{z}^{l}\right) \\
M_{x}^{l}= & 2 B_{21}^{l} \frac{\partial u_{x}^{l}}{\partial x}+2 D_{22}^{l} \frac{\partial \phi_{l}}{\partial x}+K_{M \Omega^{\prime}}^{l} \\
& +\kappa B_{12}^{l}\left(\frac{\partial u_{z}}{\partial x}+\psi_{z}^{l}\right) \\
& -\kappa\left(A_{32}^{l}-A_{23}^{l}\right)\left(\frac{\partial u_{y}}{\partial x}-\psi_{y}^{l}\right) \\
& +\kappa B_{13}^{l}\left(\frac{\partial u_{z}}{\partial x}+\psi_{z}^{l}\right) \\
M_{y}^{l}= & D_{11}^{l} \frac{\partial \psi_{z}^{l}}{\partial x}-\kappa B_{12}^{l}\left(\frac{\partial u_{y}}{\partial x}-\psi_{y}^{l}\right)
\end{aligned}
$$

where

$$
\begin{aligned}
A_{i j}^{l} & =\frac{\pi}{2} \sum_{k=1}^{n} \bar{k}_{i j}^{(k)}\left(r_{k}^{2}-r_{k-1}^{2}\right), \\
B_{i j}^{l} & =\frac{\pi}{3} \sum_{k=1}^{n} \bar{k}_{i j}^{(k)}\left(r_{k}^{3}-r_{k-1}^{3}\right), \\
D_{i j}^{l} & =\frac{\pi}{4} \sum_{k=1}^{n} \bar{k}_{i j}^{(k)}\left(r_{k}^{4}-r_{k-1}^{4}\right), \\
K_{N \Omega}^{l} & =2 \pi \sum_{k=1}^{n} \int_{r_{k-1}}^{r_{k}}\left(\bar{c}_{11}^{(k)} \sigma_{r}+\bar{c}_{12}^{(k)} \sigma_{\alpha}\right) r d r, \\
K_{M \Omega}^{l} & =2 \pi \sum_{k=1}^{n} \int_{r_{k-1}}^{r_{k}}\left(\bar{c}_{11}^{(k)} \sigma_{r}+\bar{c}_{12}^{(k)} \sigma_{\alpha}\right) r^{2} d r,
\end{aligned}
$$

$$
(l=s, c) .
$$

Taking the variation of (11) and using (7) give the following expression of the form

$$
\begin{aligned}
\delta U_{v} & =\int_{0}^{L} \int_{0}^{2 \pi} \int_{r_{c}}^{r_{s}}\left(\tau_{r x}^{v} \delta \gamma_{r x}^{v}+\tau_{x \alpha}^{v} \delta \gamma_{x \alpha}^{v}\right) r d r d \alpha d x \\
& =\int_{0}^{L} \int_{0}^{2 \pi} \int_{r_{c}}^{r_{s}}\left\{\tau_{r x}^{v}\left[\frac{\partial \delta u_{z}}{\partial x}+\frac{\left(r_{s} \delta \psi_{z}^{s}-r_{c} \delta \psi_{z}^{c}\right)}{h_{v}}\right] \sin \alpha\right. \\
& +\tau_{r x}^{v}\left[\frac{\partial \delta u_{y}}{\partial x}-\frac{\left(r_{s} \delta \psi_{y}^{s}-r_{c} \delta \psi_{y}^{c}\right)}{h_{v}}\right] \cos \alpha \\
+ & \frac{\tau_{r x}^{v}\left(\delta u_{x}^{s}-\delta u_{x}^{c}\right)}{h_{v}}+\tau_{x \alpha}^{v}\left[\frac{\partial \delta u_{z}}{\partial x}+\frac{\left(\delta \psi_{z}^{s}+\delta \psi_{z}^{c}\right)}{2}\right] \cos \alpha \\
& -\tau_{x \alpha}^{v}\left[\frac{\partial \delta u_{y}}{\partial x}-\frac{\left(\delta \psi_{y}^{s}+\delta \psi_{y}^{c}\right)}{2}\right] \sin \alpha \\
& \left.+\tau_{x \alpha}^{v}\left(r_{s} \frac{\partial \delta \phi_{s}}{\partial x}+r_{c} \frac{\partial \delta \phi_{c}}{\partial x}\right)\right\} r d r d \alpha d x
\end{aligned}
$$

The stress resultants of the viscoelastic layer have the following form:

$$
\begin{aligned}
V_{r x}^{v} & =\int_{0}^{L} \int_{0}^{2 \pi} \int_{r_{c}}^{r_{s}} \tau_{r x}^{v} r d r d \alpha d x=\frac{A_{v} G_{v}}{h_{v}}\left(u_{x}^{s}-u_{x}^{c}\right), \\
V_{x \alpha}^{v} & =\int_{0}^{L} \int_{0}^{2 \pi} \int_{r_{c}}^{r_{s}} \tau_{x \alpha}^{v} r d r d \alpha d x \\
& =\frac{A_{v} G_{v}}{2}\left(r_{s} \frac{\partial \phi_{s}}{\partial x}+r_{c} \frac{\partial \phi_{c}}{\partial x}\right), \\
V_{r x 1}^{v} & =\int_{0}^{L} \int_{0}^{2 \pi} \int_{r_{c}}^{r_{s}} \tau_{r x}^{v} r \sin \alpha d r d \alpha d x \\
& =\frac{A_{v} G_{v}}{2}\left[\frac{\partial u_{z}}{\partial x}+\frac{\left(r_{s} \psi_{z}^{s}-r_{c} \psi_{z}^{c}\right)}{h_{v}}\right],
\end{aligned}
$$




$$
\begin{aligned}
V_{r x 2}^{v} & =\int_{0}^{L} \int_{0}^{2 \pi} \int_{r_{c}}^{r_{s}} \tau_{r x}^{v} r \cos \alpha d r d \alpha d x \\
& =\frac{A_{v} G_{v}}{2}\left[\frac{\partial u_{y}}{\partial x}-\frac{\left(r_{s} \psi_{y}^{s}-r_{c} \psi_{y}^{c}\right)}{h_{v}}\right], \\
V_{x \alpha 1}^{v} & =\int_{0}^{L} \int_{0}^{2 \pi} \int_{r_{c}}^{r_{s}} \tau_{x \alpha}^{v} r \sin \alpha d r d \alpha d x \\
& =-\frac{A_{v} G_{v}}{2}\left[\frac{\partial u_{y}}{\partial x}-\frac{\left(\psi_{y}^{s}+\psi_{y}^{c}\right)}{2}\right], \\
V_{x \alpha 2}^{v} & =\int_{0}^{L} \int_{0}^{2 \pi} \int_{r_{c}}^{r_{s}} \tau_{x \alpha}^{v} r \cos \alpha d r d \alpha d x \\
& =\frac{A_{v} G_{v}}{2}\left[\frac{\partial u_{z}}{\partial x}+\frac{\left(\psi_{y}^{s}+\psi_{y}^{c}\right)}{2}\right], \\
V_{y}^{v} & =V_{r x 2}^{v}-V_{x \alpha 1}^{v}, \\
V_{z}^{v} & =V_{r x 1}^{v}+V_{x \alpha 2}^{v} .
\end{aligned}
$$

Using (18), the variation of the strain energy of the viscoelastic layer can be written as

$$
\begin{aligned}
\delta U_{v} & =\int_{0}^{L}\left[\left(V_{r x 2}^{v}-V_{x \theta 1}^{v}\right) \frac{\partial \delta u_{y}}{\partial x}+\left(V_{r x 1}^{v}+V_{x \theta 2}^{v}\right) \frac{\partial \delta u_{z}}{\partial x}\right. \\
& +\left(\frac{V_{x \theta 1}^{v}}{2}-\frac{r_{s}}{h_{v}} V_{r x 2}^{v}\right) \delta \psi_{y}^{s}+\left(\frac{V_{x \theta 1}^{v}}{2}+\frac{r_{c}}{h_{v}} V_{r x 2}^{v}\right) \delta \psi_{y}^{c} \\
& +\left(\frac{V_{x \theta 2}^{v}}{2}+\frac{r_{s}}{h_{v}} V_{r x 1}^{v}\right) \delta \psi_{z}^{s}+\left(\frac{V_{x \theta 2}^{v}}{2}-\frac{r_{c}}{h_{v}} V_{r x 1}^{v}\right) \delta \psi_{z}^{c} \\
& +\frac{V_{r x}^{v}}{h_{v}} \delta u_{x}^{s}-\frac{V_{r x}^{v}}{h_{v}} \delta u_{x}^{c}+V_{x \theta}^{v} r_{s} \frac{\partial \delta \phi_{s}}{\partial x} \\
+ & \left.V_{x \theta}^{v} r_{c} \frac{\partial \delta \phi_{c}}{\partial x}\right] d x .
\end{aligned}
$$

In order to derive the equations of the motion of the constrained layer damping shaft, the following Hamilton principle can be used:

$$
\int_{t_{1}}^{t_{2}}(\delta T-\delta U) d t=0
$$

where $\delta T=\delta T_{s}+\delta T_{c}+\delta T_{v}$ and $\delta U=\delta U_{s}+\delta U_{c}+\delta U_{v}$.

Substituting (8), (14), and (19) into (20), the equations of the motion of the shaft can be obtained as follows:

$$
\begin{aligned}
& f_{1}\left(u_{x}^{s}, u_{x}^{c}, u_{y}, u_{z}, \psi_{y}^{s}, \psi_{z}^{s}, \psi_{y}^{c}, \psi_{z}^{c}, \phi_{s}, \phi_{c}\right) \\
& \triangleq\left(m_{s}+m_{c}+m_{v}\right) \frac{\partial^{2} u_{y}}{\partial t^{2}}-\frac{\partial V_{y}^{s}}{\partial x}-\frac{\partial V_{y}^{c}}{\partial x}-\frac{\partial V_{y}^{v}}{\partial x}=0
\end{aligned}
$$

$$
\begin{aligned}
& f_{2}\left(u_{x}^{s}, u_{x}^{c}, u_{y}, u_{z}, \psi_{y}^{s}, \psi_{z}^{s}, \psi_{y}^{c}, \psi_{z}^{c}, \phi_{s}, \phi_{c}\right) \\
& \triangleq\left(m_{s}+m_{c}+m_{v}\right) \frac{\partial^{2} u_{z}}{\partial t^{2}}-\frac{\partial V_{z}^{s}}{\partial x}-\frac{\partial V_{z}^{c}}{\partial x}-\frac{\partial V_{z}^{v}}{\partial x}=0 \\
& f_{3}\left(u_{x}^{s}, u_{x}^{c}, u_{y}, u_{z}, \psi_{y}^{s}, \psi_{z}^{s}, \psi_{y}^{c}, \psi_{z}^{c}, \phi_{s}, \phi_{c}\right) \\
& \triangleq I_{s}\left(\frac{\partial^{2} \psi_{y}^{s}}{\partial t^{2}}-2 \Omega \frac{\partial \psi_{z}^{s}}{\partial t}\right)+\frac{\partial M_{z}^{s}}{\partial x}+\frac{V_{x \theta 1}^{v}}{2}-\frac{r_{s}}{h_{v}} V_{r x 2}^{v} \\
& \quad-V_{y}^{s}=0
\end{aligned}
$$$$
f_{4}\left(u_{x}^{s}, u_{x}^{c}, u_{y}, u_{z}, \psi_{y}^{s}, \psi_{z}^{s}, \psi_{y}^{c}, \psi_{z}^{c}, \phi_{s}, \phi_{c}\right)
$$$$
\triangleq I_{s}\left(\frac{\partial^{2} \psi_{z}^{s}}{\partial t^{2}}+2 \Omega \frac{\partial \psi_{y}^{s}}{\partial t}\right)-\frac{\partial M_{y}^{s}}{\partial x}+\frac{V_{x \theta 2}^{v}}{2}+\frac{r_{s}}{h_{v}} V_{r x 1}^{v}
$$$$
+V_{z}^{s}=0
$$$$
f_{5}\left(u_{x}^{s}, u_{x}^{c}, u_{y}, u_{z}, \psi_{y}^{s}, \psi_{z}^{s}, \psi_{y}^{c}, \psi_{z}^{c}, \phi_{s}, \phi_{c}\right)
$$$$
\triangleq I_{c}\left(\frac{\partial^{2} \psi_{y}^{c}}{\partial t^{2}}-2 \Omega \frac{\partial \psi_{z}^{c}}{\partial t}\right)+\frac{\partial M_{z}^{c}}{\partial x}+\frac{V_{x \theta 1}^{v}}{2}+\frac{r_{c}}{h_{v}} V_{r x 2}^{v}
$$$$
-V_{y}^{c}=0
$$$$
f_{6}\left(u_{x}^{s}, u_{x}^{c}, u_{y}, u_{z}, \psi_{y}^{s}, \psi_{z}^{s}, \psi_{y}^{c}, \psi_{z}^{c}, \phi_{s}, \phi_{c}\right)
$$$$
\triangleq I_{s}\left(\frac{\partial^{2} \psi_{z}^{c}}{\partial t^{2}}+2 \Omega \frac{\partial \psi_{y}^{c}}{\partial t}\right)-\frac{\partial M_{y}^{c}}{\partial x}+\frac{V_{x \theta 2}^{v}}{2}-\frac{r_{c}}{h_{v}} V_{r x 1}^{v}
$$$$
+V_{z}^{c}=0
$$$$
f_{7}\left(u_{x}^{s}, u_{x}^{c}, u_{y}, u_{z}, \psi_{y}^{s}, \psi_{z}^{s}, \psi_{y}^{c}, \psi_{z}^{c}, \phi_{s}, \phi_{c}\right)
$$$$
\triangleq\left(\frac{m_{v}}{4}+m_{s}\right) \frac{\partial^{2} u_{x}^{s}}{\partial t^{2}}+\frac{m_{v}}{4} \frac{\partial^{2} u_{x}^{c}}{\partial t^{2}}+\frac{V_{r x}^{v}}{h_{v}}-\frac{\partial N_{x}^{s}}{\partial x}=0
$$$$
f_{8}\left(u_{x}^{s}, u_{x}^{c}, u_{y}, u_{z}, \psi_{y}^{s}, \psi_{z}^{s}, \psi_{y}^{c}, \psi_{z}^{c}, \phi_{s}, \phi_{c}\right)
$$$$
\triangleq\left(\frac{m_{v}}{4}+m_{s}\right) \frac{\partial^{2} u_{x}^{c}}{\partial t^{2}}+\frac{m_{v}}{4} \frac{\partial^{2} u_{x}^{s}}{\partial t^{2}}-\frac{V_{r x}^{v}}{h_{v}}+\frac{\partial N_{x}^{c}}{\partial x}=0
$$$$
f_{9}\left(u_{x}^{s}, u_{x}^{c}, u_{y}, u_{z}, \psi_{y}^{s}, \psi_{z}^{s}, \psi_{y}^{c}, \psi_{z}^{c}, \phi_{s}, \phi_{c}\right)
$$$$
\triangleq\left(\frac{I_{v}}{2}+2 I_{s}\right) \frac{\partial^{2} \phi_{s}}{\partial t^{2}}+\frac{I_{v}}{2} \frac{\partial^{2} \phi_{c}}{\partial t^{2}}-\left(\frac{\partial M_{x}^{s}}{\partial x}+r_{s} \frac{\partial V_{x \theta}^{v}}{\partial x}\right)
$$$$
=0
$$$$
f_{10}\left(u_{x}^{s}, u_{x}^{c}, u_{y}, u_{z}, \psi_{y}^{s}, \psi_{z}^{s}, \psi_{y}^{c}, \psi_{z}^{c}, \phi_{s}, \phi_{c}\right)
$$$$
\triangleq\left(\frac{I_{v}}{2}+2 I_{c}\right) \frac{\partial^{2} \phi_{c}}{\partial t^{2}}+\frac{I_{v}}{2} \frac{\partial^{2} \phi_{s}}{\partial t^{2}}-\left(\frac{\partial M_{x}^{c}}{\partial x}+r_{c} \frac{\partial V_{x \theta}^{v}}{\partial x}\right)
$$$$
=0 \text {. }
$$ 
Substituting (15) and (18) into (21), the equations of motion involving variables in terms of displacements can be derived. The resulting equations can be reduced and split into two independent equation systems associated with both bending-transverse shear (see the first up to sixth equations of (21)) and extension-twist motions (see the seventh up to tenth equations of (21)). From (15) and (18), it is obvious that the extension-twist motions are not coupled to bendingtransverse shear motions. Note also that the stresses $\sigma_{r}$ and $\sigma_{\theta}$ influence only $K_{N \Omega}^{l}$ and $K_{M \Omega}^{l}$, which in turn influence only the extension-twist motions.

In present study emphasis is placed on only the problem involving the bending-transverse shear coupling.

The motion equations of bending-transverse shear coupling in terms of displacements can be shown in the form as

$$
\begin{aligned}
& f_{1}\left(u_{x}^{s}, u_{x}^{c}, u_{y}, u_{z}, \psi_{y}^{s}, \psi_{z}^{s}, \psi_{y}^{c}, \psi_{z}^{c}, \phi_{s}, \phi_{c}\right) \\
& \triangleq\left(m_{s}+m_{c}+m_{v}\right) \frac{\partial^{2} u_{y}}{\partial t^{2}} \\
&-\left[\kappa\left(A_{22}^{s}+A_{33}^{s}\right)+\kappa\left(A_{22}^{c}+A_{33}^{c}\right)+A_{v} G_{v}\right] \frac{\partial^{2} u_{y}}{\partial x^{2}} \\
&-\left[\kappa\left(A_{32}^{s}-A_{23}^{s}\right)+\kappa\left(A_{32}^{c}-A_{23}^{c}\right)\right] \frac{\partial^{2} u_{z}}{\partial x^{2}} \\
&+B_{31}^{s} \frac{\partial^{2} \psi_{y}^{s}}{\partial x^{2}}+B_{21}^{s} \frac{\partial^{2} \psi_{z}^{s}}{\partial x^{2}}+B_{31}^{c} \frac{\partial^{2} \psi_{y}^{c}}{\partial x^{2}}+B_{21}^{c} \frac{\partial^{2} \psi_{z}^{c}}{\partial x^{2}} \\
&+\left[\kappa\left(A_{22}^{s}+A_{33}^{s}\right)+A_{v} G_{v} \frac{\bar{r}_{s}}{h_{v}}\right] \frac{\partial \psi_{y}^{s}}{\partial x} \\
&+\left[\kappa\left(A_{22}^{c}+A_{33}^{c}\right)-A_{v} G_{v} \frac{\bar{r}_{c}}{h_{v}}\right] \frac{\partial \psi_{y}^{c}}{\partial x} \\
&+\kappa\left(A_{32}^{s}-A_{23}^{s}\right) \frac{\partial \psi_{z}^{s}}{\partial x}+\kappa\left(A_{32}^{c}-A_{23}^{c}\right) \frac{\partial \psi_{z}^{c}}{\partial x}=0 \\
&\left.\left.+\left[\kappa\left(A_{32}^{s}-A_{23}^{s}\right)+\kappa\left(A_{32}^{c}-A_{23}^{c}\right)\right] \frac{\partial^{2} \psi_{y}^{s}}{\partial x^{2}}-B_{31}^{s} \frac{\partial^{2} \psi_{z}^{s}}{\partial x^{2}}+B_{21}^{s}\right)+A_{v}^{c} G_{v} \frac{\partial_{s}}{\partial x_{v}^{2}}\right] \frac{\partial \psi_{z}^{s}}{\partial x} \\
& f_{2}\left(u_{x}^{s}, u_{x}^{c}, u_{y}, u_{z}, \psi_{y}^{s}, \psi_{z}^{s}, \psi_{y}^{c}, \psi_{z}^{c}, \phi_{s}, \phi_{c}\right) \\
& \triangleq\left(m_{s}+m_{c}+m_{v}\right) \frac{\partial^{2} u_{z}}{\partial t^{2}} \\
&-\left[\kappa\left(A_{22}^{s}+A_{33}^{s}\right)+\kappa\left(A_{22}^{c}+A_{33}^{c}\right)+A_{v} G_{v}\right] \frac{\partial^{2} u_{z}}{\partial x^{2}} \\
&+
\end{aligned}
$$

$$
\begin{aligned}
& -\left[\kappa\left(A_{22}^{c}+A_{33}^{c}\right)-A_{v} G_{v} \frac{\bar{r}_{c}}{h_{v}}\right] \frac{\partial \psi_{z}^{c}}{\partial x} \\
& -\kappa\left(A_{32}^{s}-A_{23}^{s}\right) \frac{\partial \psi_{y}^{s}}{\partial x}-\kappa\left(A_{32}^{c}-A_{23}^{c}\right) \frac{\partial \psi_{y}^{c}}{\partial x}=0 \\
& f_{3}\left(u_{x}^{s}, u_{x}^{c}, u_{y}, u_{z}, \psi_{y}^{s}, \psi_{z}^{s}, \psi_{y}^{c}, \psi_{z}^{c}, \phi_{s}, \phi_{c}\right) \\
& \triangleq I_{s}\left(\frac{\partial^{2} \psi_{y}^{s}}{\partial t^{2}}-2 \Omega \frac{\partial \psi_{z}^{s}}{\partial t}\right)-D_{11}^{s} \frac{\partial^{2} \psi_{y}^{s}}{\partial x^{2}}+\kappa B_{13}^{s} \frac{\partial^{2} u_{y}}{\partial x^{2}} \\
& +\left(B_{31}^{s}-\kappa B_{13}^{s}\right) \frac{\partial \psi_{y}^{s}}{\partial x} \\
& -\left[\kappa\left(A_{22}^{s}+A_{33}^{s}\right)+A_{v} G_{v} \frac{\bar{r}_{s}}{h_{v}}\right] \frac{\partial u_{y}}{\partial x} \\
& +\left[\kappa\left(A_{22}^{s}+A_{33}^{s}\right)+A_{v} G_{v} \frac{\bar{r}_{s s}^{2}}{h_{v}^{2}}\right] \psi_{y}^{s}+\kappa B_{12}^{s} \frac{\partial^{2} u_{z}}{\partial x^{2}} \\
& -\kappa\left(A_{32}^{s}-A_{23}^{s}\right) \frac{\partial u_{z}}{\partial x}+\left(B_{21}^{s}+\kappa B_{12}^{s}\right) \frac{\partial \psi_{z}^{s}}{\partial x} \\
& -G_{v} A_{v} \frac{\bar{r}_{s c}^{2}}{h_{v}^{2}} \psi_{y}^{c}-\kappa\left(A_{32}^{s}-A_{23}^{s}\right) \psi_{z}^{s}=0 \\
& f_{4}\left(u_{x}^{s}, u_{x}^{c}, u_{y}, u_{z}, \psi_{y}^{s}, \psi_{z}^{s}, \psi_{y}^{c}, \psi_{z}^{c}, \phi_{s}, \phi_{c}\right) \\
& \triangleq I_{s}\left(\frac{\partial^{2} \psi_{z}^{s}}{\partial t^{2}}+2 \Omega \frac{\partial \psi_{y}^{s}}{\partial t}\right)-D_{11}^{s} \frac{\partial^{2} \psi_{z}^{s}}{\partial x^{2}}-\kappa B_{13}^{s} \frac{\partial^{2} u_{z}}{\partial x^{2}} \\
& +\left(B_{31}^{s}-\kappa B_{13}^{s}\right) \frac{\partial \psi_{z}^{s}}{\partial x} \\
& +\left[\kappa\left(A_{22}^{s}+A_{33}^{s}\right)+A_{v} G_{v} \frac{\bar{r}_{s}}{h_{v}}\right] \frac{\partial u_{z}}{\partial x} \\
& +\left[\kappa\left(A_{22}^{s}+A_{33}^{s}\right)+A_{v} G_{v} \frac{\bar{r}_{s s}^{2}}{h_{v}^{2}}\right] \psi_{z}^{s}+\kappa B_{12}^{s} \frac{\partial^{2} u_{y}}{\partial x^{2}} \\
& -\kappa\left(A_{32}^{s}-A_{23}^{s}\right) \frac{\partial u_{y}}{\partial x}-\left(B_{21}^{s}+\kappa B_{12}^{s}\right) \frac{\partial \psi_{y}^{s}}{\partial x} \\
& -G_{v} A_{v} \frac{\bar{r}_{s c}^{2}}{h_{v}^{2}} \psi_{z}^{c}+\kappa\left(A_{32}^{s}-A_{23}^{s}\right) \psi_{y}^{s}=0, \\
& f_{5}\left(u_{x}^{s}, u_{x}^{c}, u_{y}, u_{z}, \psi_{y}^{s}, \psi_{z}^{s}, \psi_{y}^{c}, \psi_{z}^{c}, \phi_{s}, \phi_{c}\right) \\
& \triangleq I_{c}\left(\frac{\partial^{2} \psi_{y}^{c}}{\partial t^{2}}-2 \Omega \frac{\partial \psi_{z}^{c}}{\partial t}\right)-D_{11}^{c} \frac{\partial^{2} \psi_{y}^{c}}{\partial x^{2}}+\kappa B_{13}^{c} \frac{\partial^{2} u_{y}}{\partial x^{2}} \\
& +\left(B_{31}^{c}-\kappa B_{13}^{c}\right) \frac{\partial \psi_{y}^{c}}{\partial x} \\
& +k_{8}\left[-\kappa\left(A_{22}^{c}+A_{33}^{c}\right)+A_{v} G_{v} \frac{\bar{r}_{c}}{h_{v}}\right] \frac{\partial u_{y}}{\partial x} \\
& +\left[\kappa\left(A_{22}^{c}+A_{33}^{c}\right)+A_{v} G_{v} \frac{\bar{r}_{c c}^{2}}{h_{v}^{2}}\right] \psi_{y}^{c}+\kappa B_{12}^{c} \frac{\partial^{2} u_{z}}{\partial x^{2}}
\end{aligned}
$$




$$
\begin{aligned}
&-\kappa\left(A_{32}^{c}-A_{23}^{c}\right) \frac{\partial u_{z}}{\partial x}+\left(B_{21}^{c}+\kappa B_{12}^{c}\right) \frac{\partial \psi_{z}^{c}}{\partial x} \\
&-G_{v} A_{v} \frac{\bar{r}_{s c}^{2}}{h_{v}^{2}} \psi_{y}^{s}-\kappa\left(A_{32}^{c}-A_{23}^{c}\right) \psi_{z}^{c}=0, \\
& f_{6}\left(u_{x}^{s}, u_{x}^{c}, u_{y}, u_{z}, \psi_{y}^{s}, \psi_{z}^{s}, \psi_{y}^{c}, \psi_{z}^{c}, \phi_{s}, \phi_{c}\right) \\
& \triangleq I_{c}\left(\frac{\partial^{2} \psi_{z}^{c}}{\partial t^{2}}+2 \Omega \frac{\partial \psi_{y}^{c}}{\partial t}\right)-D_{11}^{c} \frac{\partial^{2} \psi_{z}^{c}}{\partial x^{2}}-\kappa B_{13}^{c} \frac{\partial^{2} u_{z}}{\partial x^{2}} \\
&+\left(B_{31}^{c}-\kappa B_{13}^{c}\right) \frac{\partial \psi_{z}^{c}}{\partial x} \\
&-\left[-\kappa\left(A_{22}^{c}+A_{33}^{c}\right)+A_{v} G_{v} \frac{\bar{r}_{c}}{h_{v}}\right] \frac{\partial u_{z}}{\partial x} \\
&+\left[\kappa\left(A_{22}^{c}+A_{33}^{c}\right)+A_{v} G_{v} \frac{\bar{r}_{c c}^{2}}{h_{v}^{2}}\right] \psi_{z}^{c}+\kappa B_{12}^{c} \frac{\partial^{2} u_{y}}{\partial x^{2}} \\
& \quad-\kappa\left(A_{32}^{c}-A_{23}^{c}\right) \frac{\partial u_{y}}{\partial x}-\left(B_{21}^{c}+\kappa B_{12}^{c}\right) \frac{\partial \psi_{y}^{c}}{\partial x} \\
& \quad-G_{v} A_{v} \frac{\bar{r}_{s c}^{2}}{h_{v}^{2}} \psi_{z}^{s}+\kappa\left(A_{32}^{c}-A_{23}^{c}\right) \psi_{y}^{c}=0,
\end{aligned}
$$

where $\bar{r}_{s}=\left(r_{s}+h_{v} / 2\right) / 2, \bar{r}_{c}=\left(r_{c}-h_{v} / 2\right) / 2, \bar{r}_{s s}^{2}=\left(r_{s}^{2}+h_{v}^{2} / 4\right) / 2$, $\bar{r}_{c c}^{2}=\left(r_{c}^{2}+h_{v}^{2} / 4\right) / 2$, and $\bar{r}_{s c}^{2}=\left(r_{s} r_{c}-h_{v}^{2} / 4\right) / 2$.

The shaft is assumed to be the simply supported boundary conditions for which there are

$$
\begin{aligned}
u_{y}(0) & =u_{z}(0)=0 \\
u_{y}(L) & =u_{z}(L)=0 \\
M_{y}^{s}(0) & =M_{y}^{c}(0)=M_{z}^{s}(0)=M_{z}^{c}(0)=0 \\
M_{y}^{s}(L) & =M_{y}^{c}(L)=M_{z}^{s}(L)=M_{z}^{c}(L)=0 .
\end{aligned}
$$

2.5. General Galerkin Method. In order to find the approximate solution of the rotating composite shaft, the quantities $u_{y}(x, t), u_{z}(x, t), \psi_{y}^{s}(x, t), \psi_{z}^{s}(x, t), \psi_{y}^{c}(x, t)$, and $\psi_{z}^{c}(x, t)$ are assumed in the form

$$
\begin{aligned}
& u_{y}(x, t)=\sum_{j=1}^{N} \xi_{j}(x) U_{y j}(t) \\
& u_{z}(x, t)=\sum_{j=1}^{N} \xi_{j}(x) U_{z j}(t) \\
& \psi_{y}^{s}(x, t)=\sum_{j=1}^{N} \eta_{j}^{s}(x) \Phi_{y j}^{s}(t) \\
& \psi_{z}^{s}(x, t)=\sum_{j=1}^{N} \eta_{j}^{s}(x) \Phi_{z j}^{s}(t)
\end{aligned}
$$

$$
\begin{aligned}
& \psi_{y}^{c}(x, t)=\sum_{j=1}^{N} \eta_{j}^{c}(x) \Phi_{y j}^{c}(t) \\
& \psi_{z}^{c}(x, t)=\sum_{j=1}^{N} \eta_{j}^{c}(x) \Phi_{z j}^{c}(t),
\end{aligned}
$$

where $U_{y j}(t), U_{z j}(t), \Phi_{y j}^{s}(t), \Phi_{z j}^{s}(t), \Phi_{y j}^{c}(t)$, and $\Phi_{z j}^{c}(t)$ are the generalized coordinates and $\xi_{j}(x), \eta_{j}^{s}(x)$, and $\eta_{j}^{c}(x)$ are the assumed mode shape functions, which are taken to be

$$
\begin{aligned}
& \xi_{j}(x)=\sin \left(\frac{j \pi x}{L}\right) \\
& \eta_{j}^{s}(x)=\eta_{j}^{c}(x)=\cos \left(\frac{j \pi x}{L}\right) .
\end{aligned}
$$

Note that the mode shape functions $\xi_{j}(x)$ satisfy the geometric boundary conditions of the shaft, as given by (23). However, the mode shape functions $\eta_{j}^{s}(x)$ and $\eta_{j}^{c}(x)$ do not satisfy the boundary conditions given by (24). In order to approximately satisfy the boundary conditions, the general Galerkin method [2] is used. The method consists of substituting approximate solutions (25) in the equations of motion (22) and multiplying these by the mode shape functions and integrating from 0 to $L$, which gives differential equation residuals. Furthermore, the boundary conditions (24) are handled by substituting (25) into (24) and then multiplying by the associated boundary values of the mode shape functions and then the boundary residuals can be obtained. By letting the sum of the differential equation and boundary residuals be equal to zero and integrating by parts, the discretized equations of the problem can be written as

$$
\begin{gathered}
\int_{0}^{L} f_{1}\left[\ldots, u_{y}(x, t), u_{z}(x, t), \ldots\right] \xi_{i}(x) d x=0, \\
i=1,2, \ldots, N \\
\int_{0}^{L} f_{2}\left[\ldots, u_{y}(x, t), u_{z}(x, t), \ldots\right] \xi_{i}(x) d x=0, \\
i=1,2, \ldots, N \\
\int_{0}^{L} f_{3}\left[\ldots, u_{y}(x, t), u_{z}(x, t), \ldots\right] \eta_{i}(x) d x \\
-\eta_{i}(L) M_{z}^{s}(L, t)+\eta_{i}(0) M_{z}^{s}(0, t)=0, \\
i=1,2, \ldots, N \\
\int_{0}^{L} f_{4}\left[\ldots, u_{y}(x, t), u_{z}(x, t), \ldots\right] \eta_{i}(x) d x \\
+\eta_{i}(L) M_{y}^{s}(L, t)-\eta_{i}(0) M_{y}^{s}(0, t)=0,
\end{gathered}
$$




$$
\begin{gathered}
\int_{0}^{L} f_{5}\left[\ldots, u_{y}(x, t), u_{z}(x, t), \ldots\right] \eta_{i}(x) d x \\
-\eta_{i}(L) M_{z}^{c}(L, t)+\eta_{i}(0) M_{z}^{c}(0, t)=0, \\
i=1,2, \ldots, N \\
\int_{0}^{L} f_{6}\left[\ldots, u_{y}(x, t), u_{z}(x, t), \ldots\right] \eta_{i}(x) d x \\
+\eta_{i}(L) M_{y}^{c}(L, t)-\eta_{i}(0) M_{y}^{c}(0, t)=0, \\
i=1,2, \ldots, N .
\end{gathered}
$$

From (27), the following ordinary equations in matrix form can be found:

$$
[M]\left\{\begin{array}{l}
\ddot{\mathbf{U}}_{y j} \\
\ddot{\mathbf{U}}_{z j} \\
\ddot{\boldsymbol{\Phi}}_{y j}^{s} \\
\ddot{\boldsymbol{\Phi}}_{z j}^{s} \\
\ddot{\boldsymbol{\Phi}}_{y j}^{c} \\
\ddot{\boldsymbol{\Phi}}_{z j}^{c}
\end{array}\right\}+[G]\left\{\begin{array}{c}
\dot{\mathbf{U}}_{y j} \\
\dot{\mathbf{U}}_{z j} \\
\dot{\boldsymbol{\Phi}}_{y j}^{s} \\
\dot{\boldsymbol{\Phi}}_{z j}^{s} \\
\dot{\boldsymbol{\Phi}}_{y j}^{c} \\
\dot{\boldsymbol{\Phi}}_{z j}^{c}
\end{array}\right\}+[K]\left\{\begin{array}{l}
\mathbf{U}_{y j} \\
\mathbf{U}_{z j} \\
\boldsymbol{\Phi}_{y j}^{s} \\
\boldsymbol{\Phi}_{z j}^{s} \\
\boldsymbol{\Phi}_{y j}^{c} \\
\boldsymbol{\Phi}_{z j}^{c}
\end{array}\right\}=\left\{\begin{array}{l}
\mathbf{0} \\
\mathbf{0} \\
\mathbf{0} \\
\mathbf{0} \\
\mathbf{0} \\
\mathbf{0}
\end{array}\right\},
$$

where $[M],[G]$, and $[K]$ denote the mass, gyroscopic, and stiffness matrix, respectively. The detailed expressions of these matrices are given in Appendix A.

\section{Numerical Results and Discussion}

In the first example, we investigate the composite shaft considered by Zinberg and Symmonds. The material properties of each ply are $L=2.47 \mathrm{~m}$, mean radius $=0.0635 \mathrm{~m}$, wall thickness $t=0.1321 \times 10^{-3} \mathrm{~m}, 10$ layers of equal thickness from innermost $\left[90^{\circ} / 45^{\circ} /-45^{\circ} /\left[0^{\circ}\right]_{6} / 90^{\circ}\right], \rho=1965 \mathrm{~kg} / \mathrm{m}^{3}, E_{11}$ $=211 \mathrm{Gpa}, E_{22}=24.1 \mathrm{Gpa}, G_{12}=6.9 \mathrm{Gpa}$, and $\nu_{12}=0.36$. The critical speed obtained by different investigators using various theories is given in Table 1. The result obtained using present model is also shown in this table. As can be seen from Table 1 the present result is close to those obtained by other composite beam theories. From this table, it can be seen that to obtain an accurate result no more than 9 mode shape functions are required. This indicates clearly that the convergence of the present model is quite good. Based on mode convergence examination, it is found that $N=9$ gives suitably converged eigenvalues. So for all results given in this paper $N=9$ unless otherwise noted.

Next, a thin tube made from a single layer which is considered by Singh and Gupta [5] is investigated. The tube parameters are as follows: $L=1.0 \mathrm{~m}$, mean radius $=0.05 \mathrm{~m}$, wall thickness $t=1 \mathrm{~mm}, E_{11}=130 \mathrm{Gpa}, E_{22}=10 \mathrm{Gpa}, G_{12}$ $=7 \mathrm{Gpa}, \nu_{12}=0.25$, and $\rho=1500 \mathrm{~kg} / \mathrm{m}^{3}$. Table 2 gives the frequencies of the thin-walled tube calculated for different fiber angles. From the table it is obvious that the results predicted by the present model are very close to those of Singh and Gupta [5] for the fiber angle $\theta$ from $0^{\circ}$ to $90^{\circ}$.
In the following example, the geometry and material properties of the composite shaft are as follows: $L=1 \mathrm{~m}$, mean radius $=0.05 \mathrm{~m}$, wall thickness $t=4 \times 10^{-3} \mathrm{~m}$, 10 layers of equal thickness with lamination $[\theta /-\theta]_{5}, \rho=1578 \mathrm{~kg} / \mathrm{m}^{3}$, $E_{11}=139 \mathrm{Gpa}, E_{22}=11 \mathrm{Gpa}, G_{12}=G_{13}=6.05 \mathrm{Gpa}, G_{23}=$ $3.78 \mathrm{Gpa}$, and $v_{12}=0.313$. The material properties of the constraining layer are assumed to be the same as the shaft. The geometry and material properties of the constrained layer are as follows: the thickness $2 \times 10^{-3} \mathrm{~m}$, the complex shear modulus $G_{v}=G(1+i \eta)=69(1+i) \mathrm{Mpa}$, and $\rho_{v}=$ $1575 \mathrm{kgm}^{-3}$. For all results given in this paper the above parameters are used unless otherwise noted.

Figures 2(a)-2(c) show the first three natural frequencies versus fiber angle for various thicknesses of constraining layer. From these figures it is obvious that the fiber angle has significant influence on the natural frequencies of the shaft. As the fiber angle increases, the natural frequencies initially increase and then decrease. The fiber angles where the highest frequency exits are $15^{\circ}, 25^{\circ}$, and $30^{\circ}$ for the first, second, and third mode, respectively. Moreover, as the results reveal, with $h_{c}$ increasing, the natural frequencies of the shaft increase.

Figures 3(a)-3(c) show the first three modal dampings versus fiber angle for various thicknesses of constraining layer. The results show that as the ply angle increases, the modal dampings are successively falling and rising. It can also be noted that the modal damping is highest when the beam has the fiber-orientation of $\theta=0^{\circ}$. The modal dampings increase as the thickness of constraining layer for the fiber angle in the range $0^{\circ}-90^{\circ}$. Similar conclusion has been arrived at by [24]. It seems that the modal dampings are more likely to be affected by the thickness of constraining layer for the fiber angle in the range $15^{\circ}-35^{\circ}$.

Figures 4(a)-4(c) show the first three natural frequencies versus fiber angle for various thicknesses of viscoelastic damping layer. It can be observed that the natural frequencies tend to decrease with the increase of the thickness of viscoelastic damping layer. This means that thickness of viscoelastic damping layer has opposite effect on the natural frequencies compared with that of constraining layer.

Figures 5(a)-5(c) show the first three modal dampings versus fiber angle for various thicknesses of viscoelastic damping layer. The results show that modal dampings increase as the thickness of viscoelastic damping layer increases. As seen in these figures, the effect of the thickness of viscoelastic damping layer on the modal dampings is similar to that previously presented for the constraining layer. Obviously, at $0^{\circ}$ fiber angle, the effect of the thickness of viscoelastic damping layer on the modal dampings is maximum.

Figures 6(a)-6(c) show the first three natural frequencies versus rotating speed for various thicknesses of constraining layer.

From these figures it can be seen that as $\Omega=0$ a single nonrotating natural frequency is obtained. When $\Omega$ is increased, due to the gyroscopic effect, the natural frequencies "split" into the forward and backward frequencies branches. It is also seen that with the increase of thickness of constraining layer the nonrotating natural 
TABLE 1: Comparison of critical speed (rpm) for composite shaft.

\begin{tabular}{|c|c|c|c|c|c|c|c|}
\hline $\begin{array}{l}\text { Reference [1] } \\
\text { (theoretical) }\end{array}$ & $\begin{array}{c}\text { Reference [1] } \\
\text { (experimental) }\end{array}$ & Reference [2] & Reference [3] & Reference [5] & Reference [6] & Reference [8] & $\begin{array}{l}\text { Present } \\
(N=9)\end{array}$ \\
\hline 5780 & 5500 & 4942 & 5762 & 5620 & 5555 & 5435 & 5619.6 \\
\hline
\end{tabular}

TABLE 2: Comparison of natural frequencies $(\mathrm{Hz})$ for composite thin-walled tube.

\begin{tabular}{lcccccc}
\hline$\theta$ (degree) & 1st & 2nd & 1st, EMBT [5] & 2nd, EMBT [5] & 1st, LBT [5] & 2nd, LBT [5] \\
\hline 0 & 426.81 & 1224.92 & 426.92 & 1225 & 426.92 & 1224.9 \\
15 & 429.84 & 1404.43 & 429.95 & 1404.5 & 429.95 & 1404.5 \\
30 & 324.99 & 1203.21 & 325.11 & 1203.3 & 832.61 & 216.62 \\
45 & 216.52 & 832.52 & 216.62 & 638.65 & 165.18 & 832.61 \\
60 & 164.95 & 638.56 & 165.17 & 558.95 & 638.66 \\
75 & 145.69 & 558.88 & 145.79 & 527.04 & 145.8 & 558.95 \\
90 & 139.94 & 526.95 & 140.13 & 527.04 \\
\hline
\end{tabular}

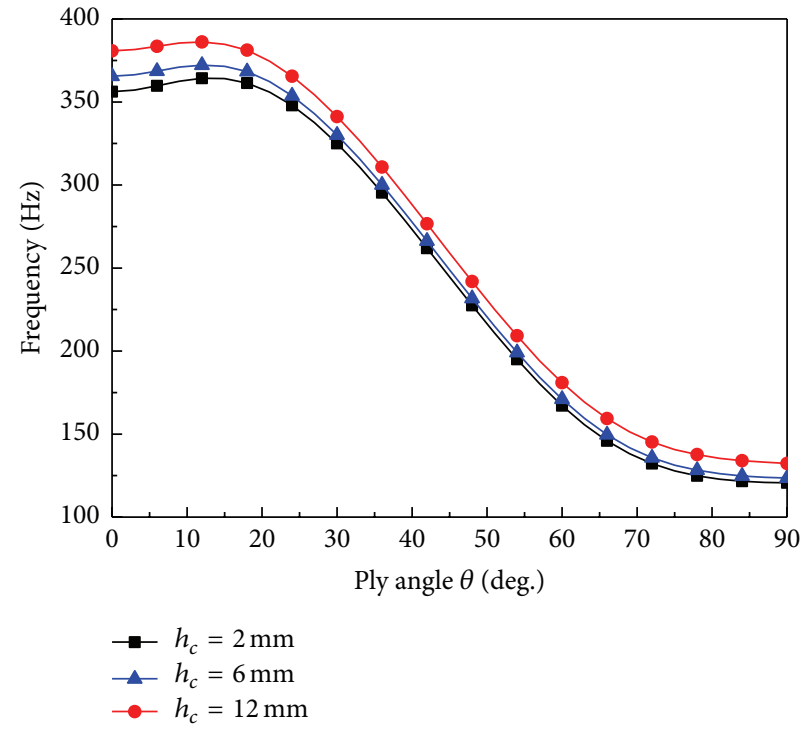

(a) First mode

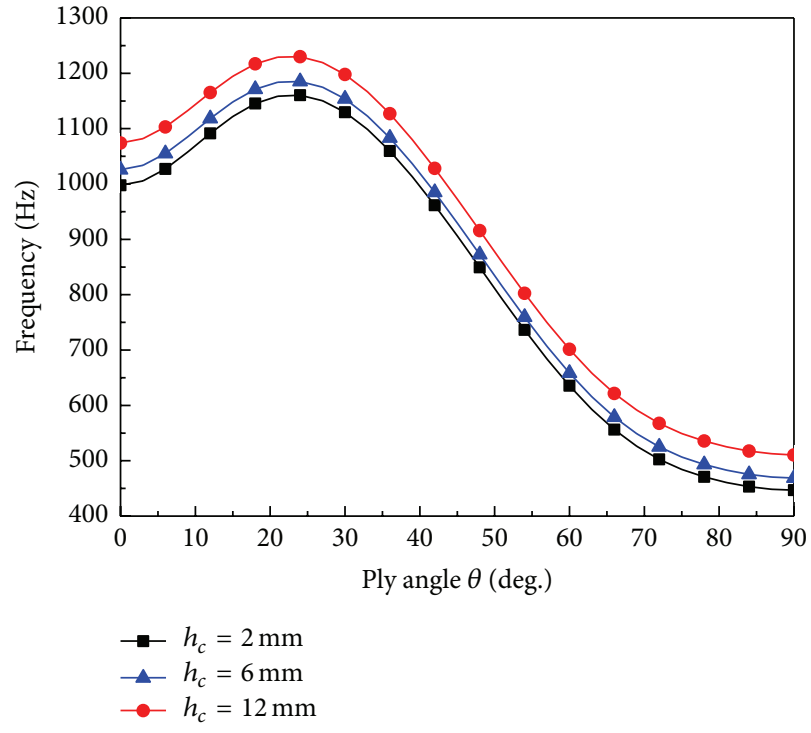

(b) Second mode

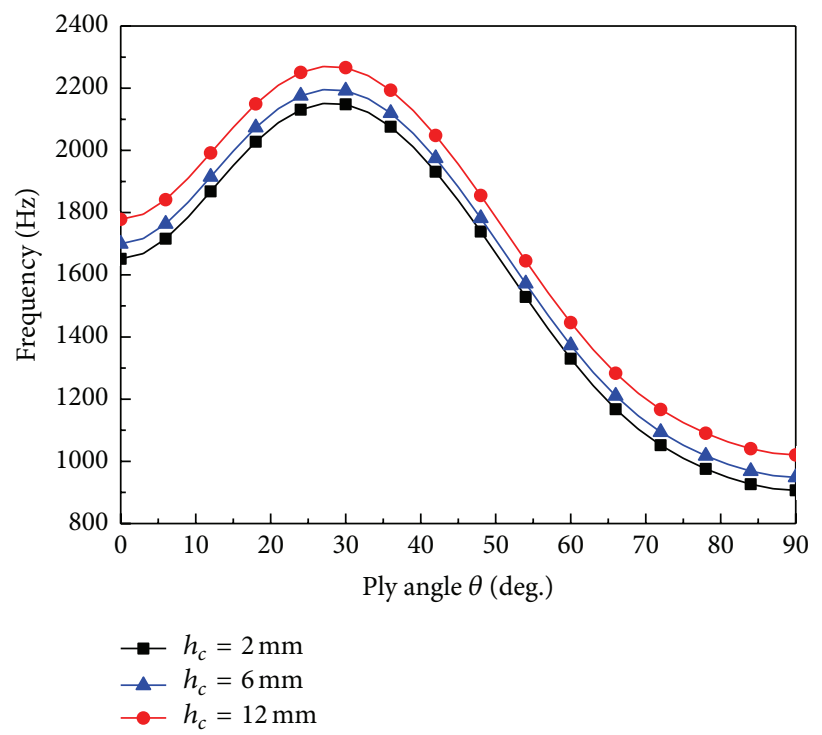

(c) Third mode

FIGURE 2: The first three natural frequencies versus fiber angle for various thicknesses of constraining layer $\left(h_{v}=2 \mathrm{~mm}, \Omega=0\right)$. 


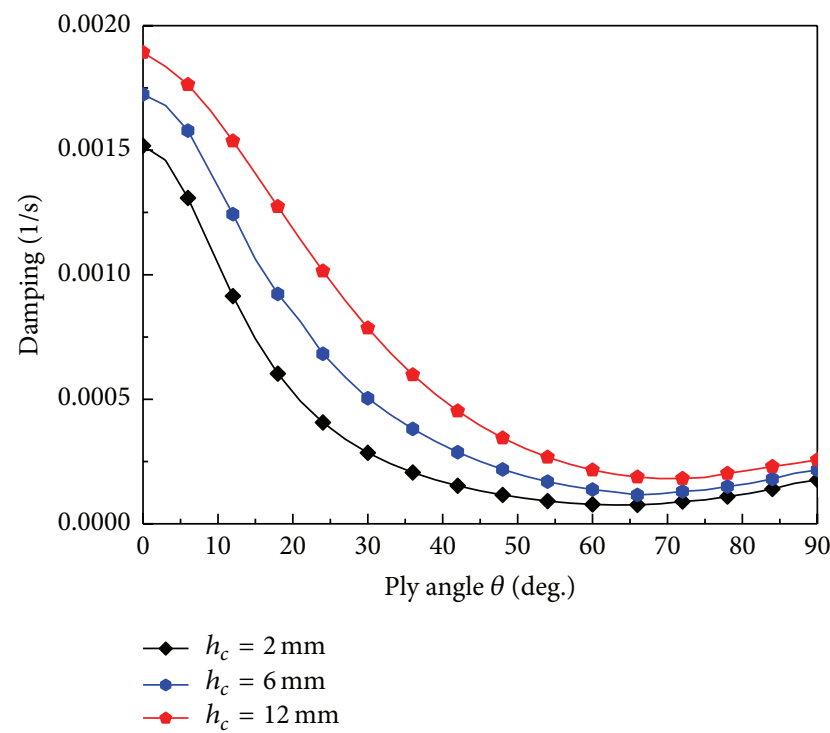

(a) First mode

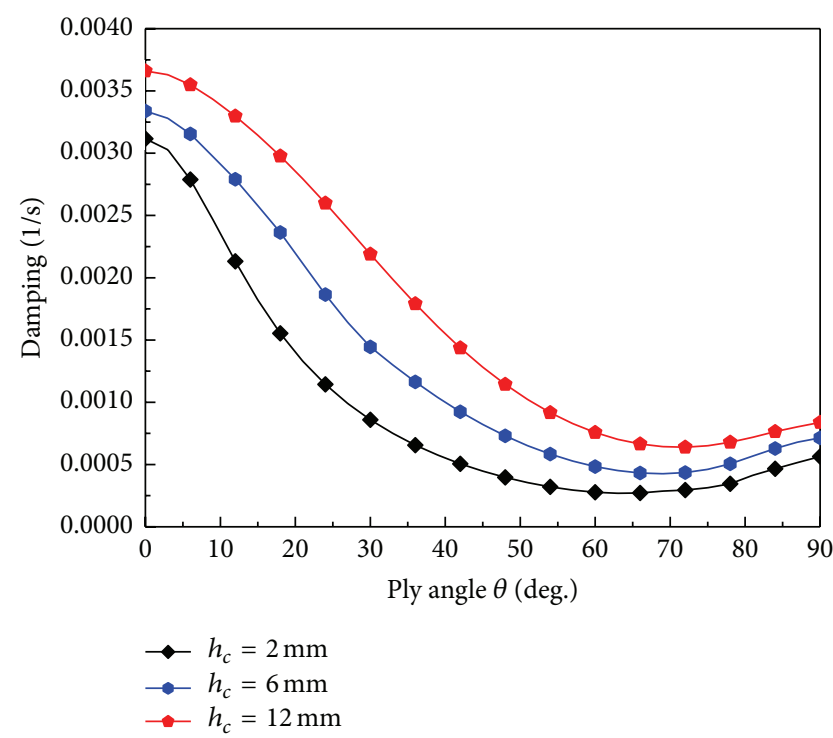

(b) Second mode

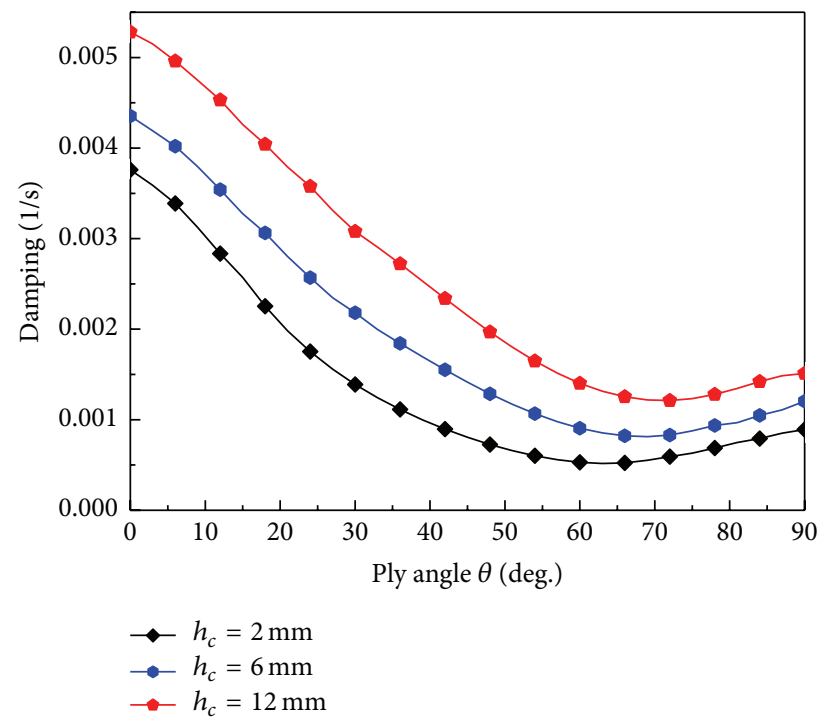

(c) Third mode

FIgURE 3: The first three dampings versus fiber angle for various thicknesses of constraining layer $\left(h_{v}=2 \mathrm{~mm}, \Omega=0\right)$.

frequencies increase. The significant effects of the thicknesses of constraining layer on the natural frequencies are observed again in these figures. These effects are consistent with that presented in Figures 2(a)-2(c). Besides, the effect of rotation on the natural frequencies is apparently weakened as the thickness of constraining layer increases.

Figures $7(a)-7(\mathrm{c})$ show the first three dampings versus rotating speed for various thicknesses of constraining layer.

As shown in these figures, the thicknesses of constraining layer have a clear influence on the modal dampings. The variation trends of the modal dampings with thicknesses of constraining layer are similar to that presented in Figures $3(\mathrm{a})-3(\mathrm{c})$. At the same time, it can also be noted that the modal dampings have the same branching phenomenon as in the natural frequencies. In addition, with the decreasing of thickness of constraining layer, the effect of rotation on the modal dampings is apparently weakened.

The effects of the thickness of viscoelastic damping layer on the curves of natural frequencies and modal dampings are presented in Figures 8 and 9, respectively. From these figures, it can be seen that the thickness of viscoelastic damping layer influences the natural frequencies and modal dampings significantly. From Figures 9(a)-9(c), it can be observed that the effects of rotation on the modal dampings tend to weaken as the thickness of viscoelastic damping layer decreases. This is similar to that previously presented for the constraining layer. However, from Figures $8(a)-8(c)$ it is also evident that 


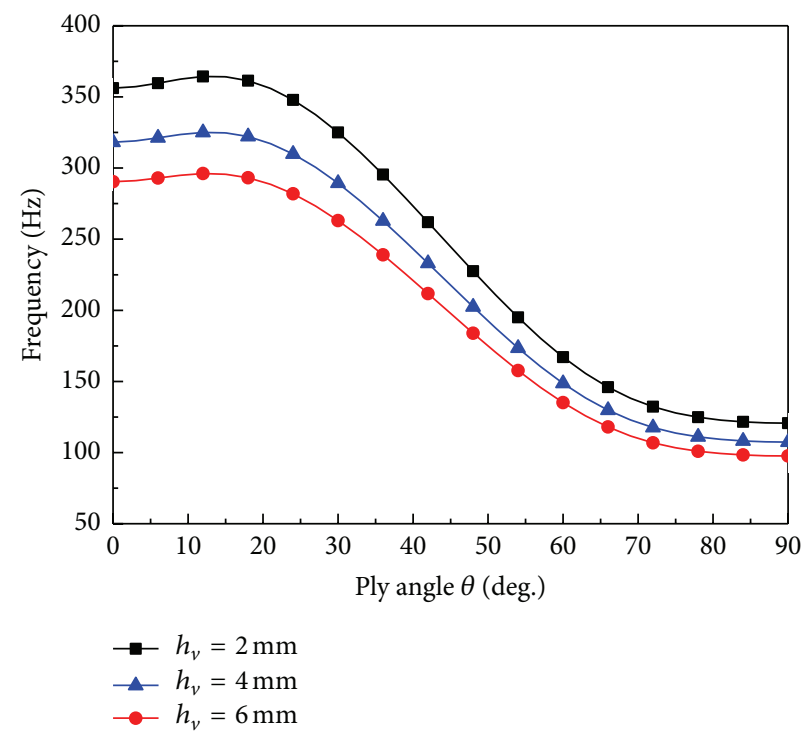

(a) First mode

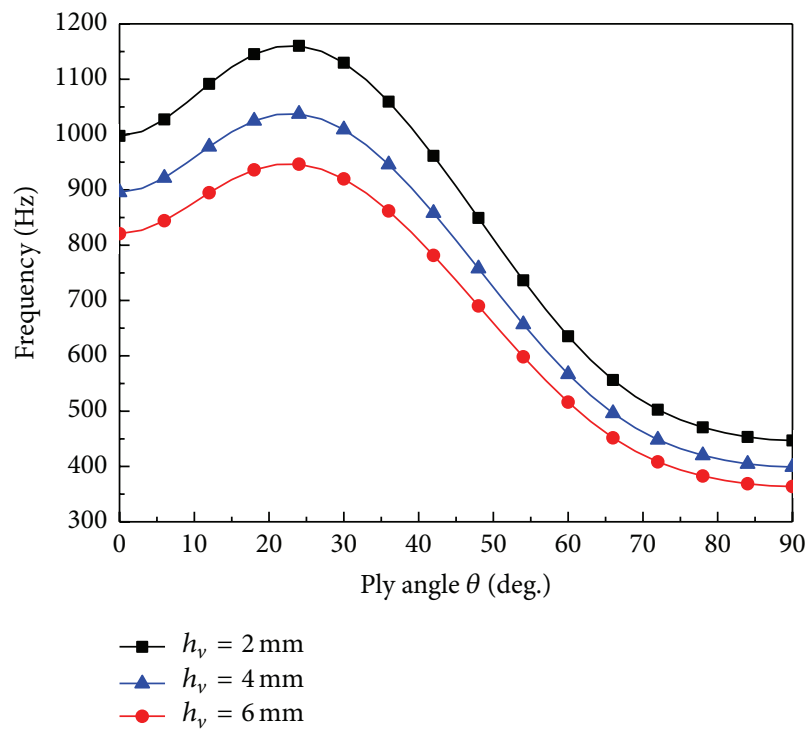

(b) Second mode

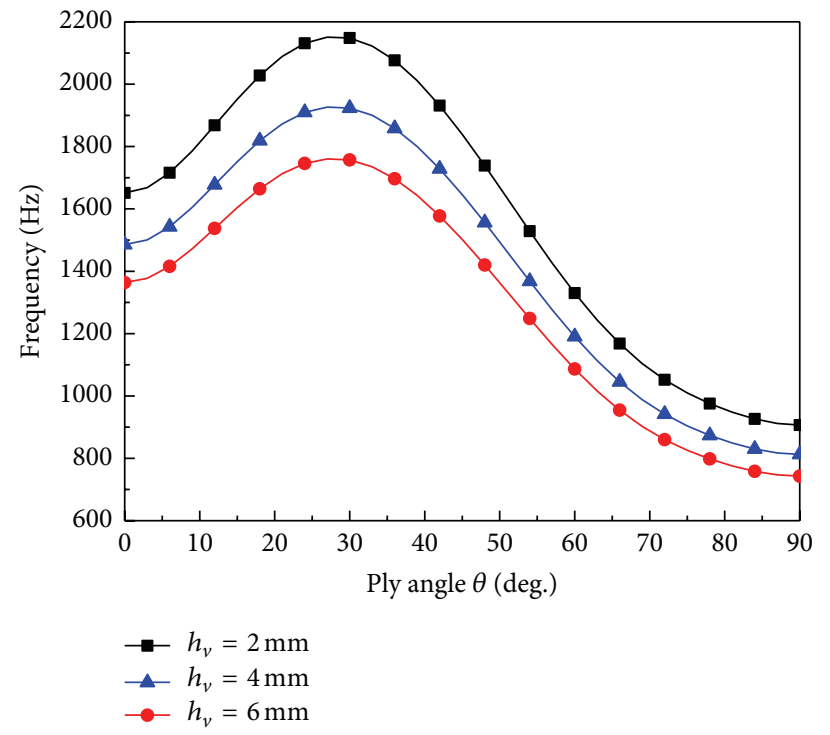

(c) Third mode

Figure 4: The first three natural frequencies versus fiber angle for various thicknesses of viscoelastic damping layer $\left(h_{c}=2 \mathrm{~mm}, \Omega=0\right)$.

the effect of rotation on the natural frequencies is significant for thin viscoelastic damping layer.

Figures 10 and 11 show the effect of the thickness of constraining layer and the thickness of viscoelastic damping layer on the curves of the critical speed versus fiber angle, respectively. The results demonstrate that thick constraining layer can raise the critical speeds while thick viscoelastic damping layer can reduce the critical speeds.

\section{Conclusions}

Based on first-order shear deformation theory of composite beam in conjunction with Hamilton's principle and general
Galerkin's method, the free vibration equations of the composite rotating shaft with CLD treatment are derived and used to predict the free vibration and damping capacity of the rotating CLD composite shafts. The present numerical result is compatible with those available in the literature. The frequencies and modal dampings are obtained using the present model. From the present analysis and the numerical results, the following main conclusions were drawn:

(1) The natural frequencies and modal dampings of the rotating CLD composite shafts increase with the thickness of constraining layer. 


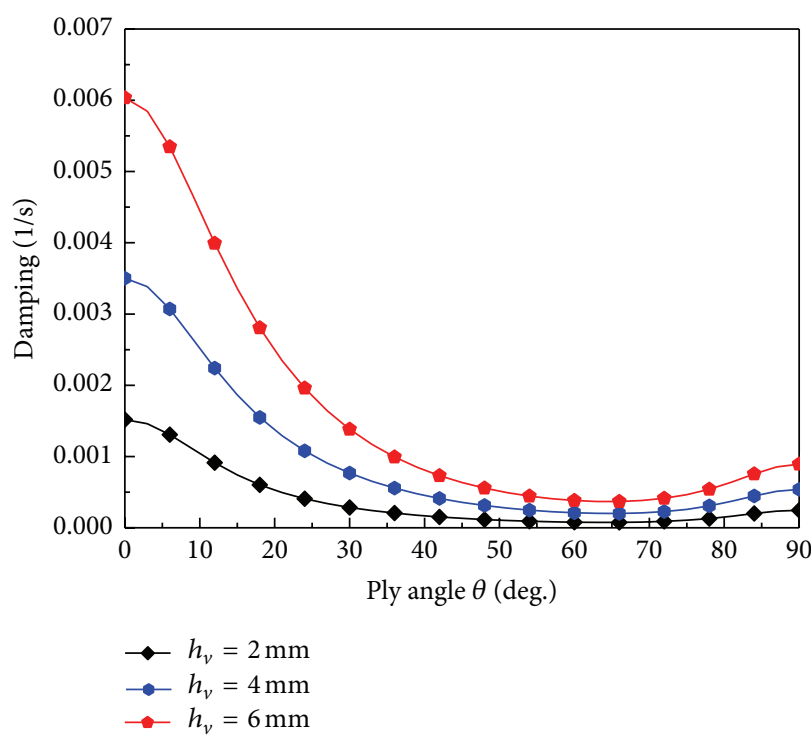

(a) First mode

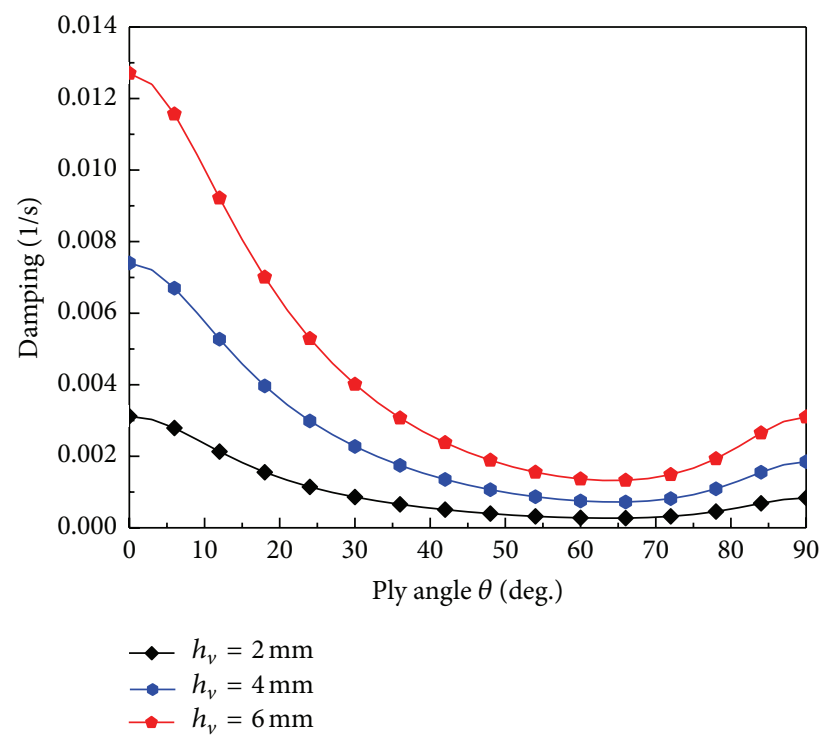

(b) Second mode

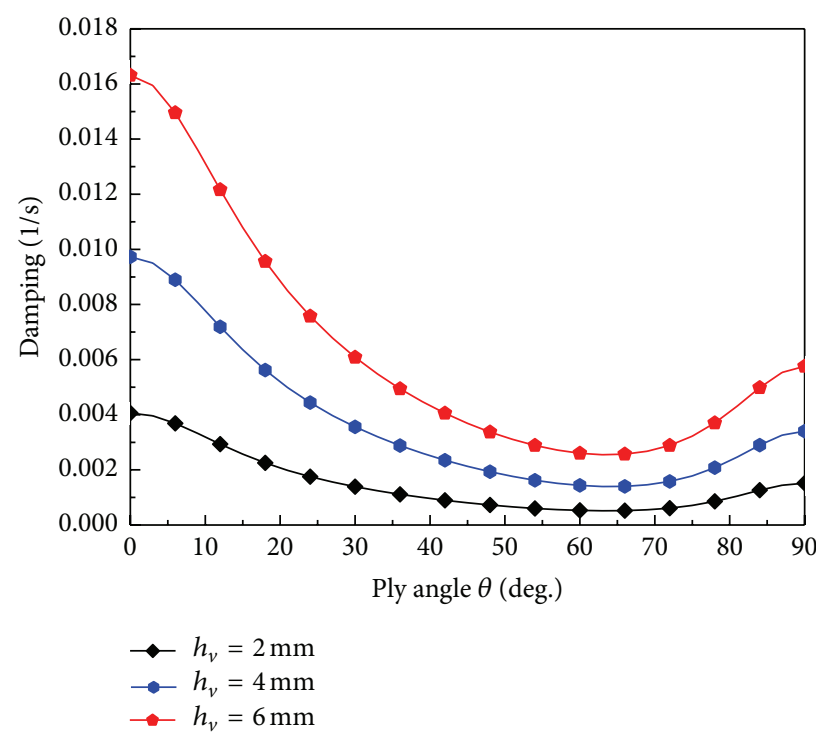

(c) Third mode

FIGURE 5: The first three dampings versus fiber angle for various thicknesses of viscoelastic damping layer $\left(h_{c}=2 \mathrm{~mm}, \Omega=0\right)$.

(2) As the thickness of viscoelastic damping layer increases, the natural frequencies decrease and the modal dampings increase.

(3) The modal dampings are more likely to be affected by constraining layer in a certain variation range of $\theta$. At $0^{\circ}$ fiber angle, the modal dampings, along with the effect of the thickness of viscoelastic damping, are maximum.

(4) The modal dampings have same branching phenomenon as in the natural frequencies due to rotation effect. The effect of rotation on the modal dampings is insignificant for thin viscoelastic damping layer or thin constraining layer.

\section{Appendix}

\section{A. The Mass, Gyroscopic, and Stiffness Matrices}

The mass, gyroscopic, and stiffness matrices in (28) are as follows:

$$
[M]=\left[\begin{array}{llllll}
\mathbf{M}_{1,1} & & & & & \\
& \mathbf{M}_{2,2} & & & & \\
& & \mathbf{M}_{3,3} & & & \\
& & & \mathbf{M}_{4,4} & & \\
& & & & \mathbf{M}_{5,5} & \\
& & & & & \mathbf{M}_{6,6}
\end{array}\right]_{6 N \times 6 N}
$$



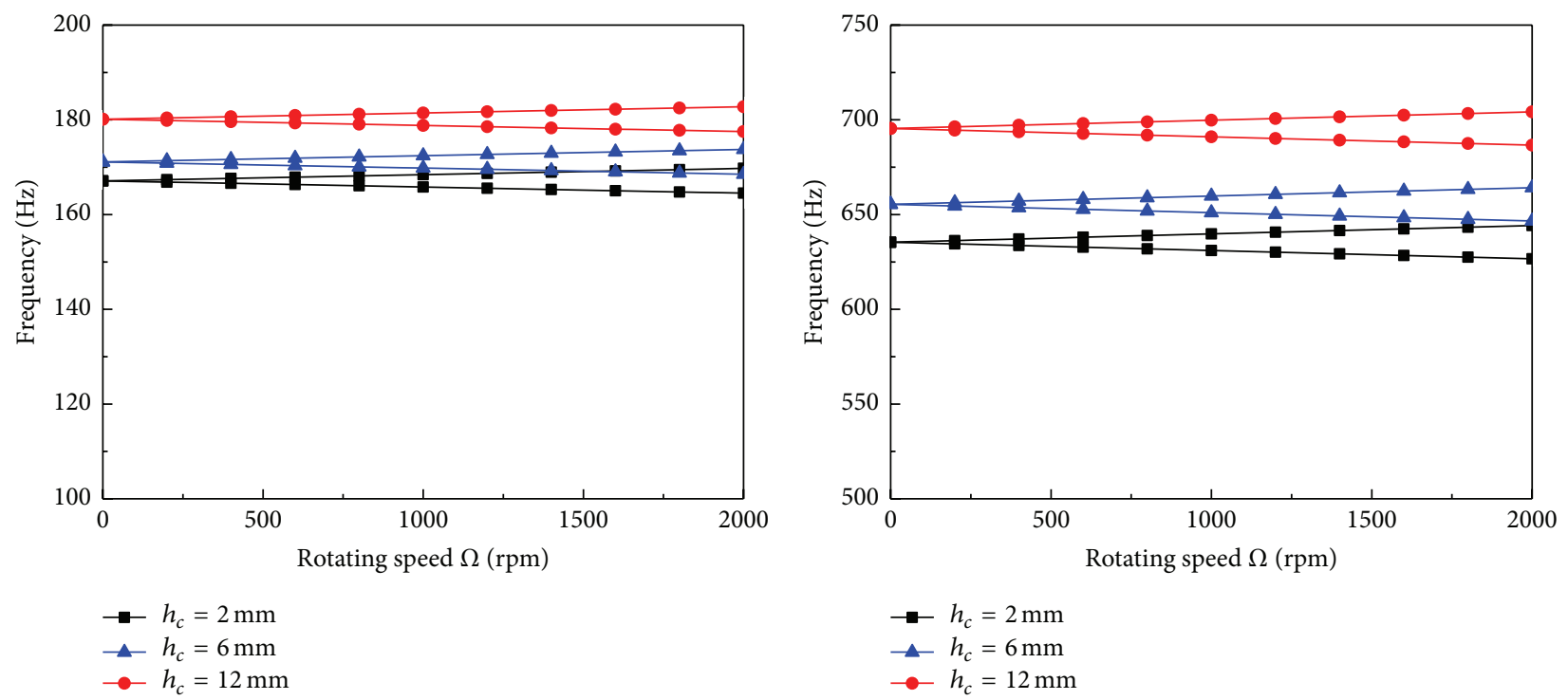

(a) First mode

(b) Second mode

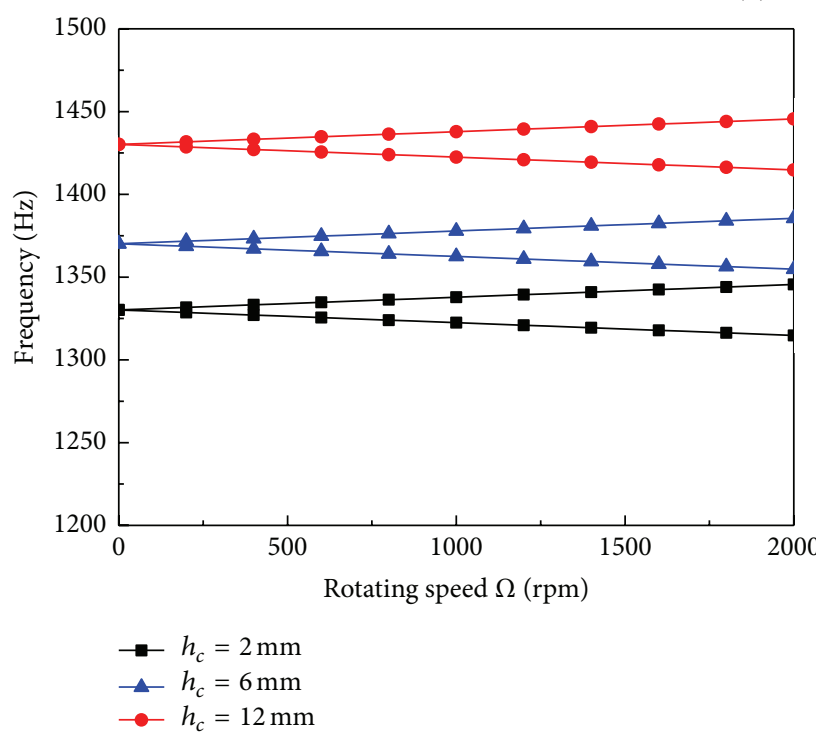

(c) Third mode

Figure 6: The first three natural frequencies versus rotating speed for various thicknesses of constraining layer $\left(h_{v}=2 \mathrm{~mm}, \theta=60^{\circ}\right)$.
$[G]=\left[\begin{array}{cccccc}\mathbf{0} & & & & & \\ & \mathbf{0} & & & & \\ & & \mathbf{0} & \mathbf{G}_{3,4} & & \\ & \mathbf{G}_{4,3} & \mathbf{0} & & \\ & & & \mathbf{0} & \mathbf{G}_{5,6} \\ & & & \mathbf{G}_{6,5} & \mathbf{0}\end{array}\right]_{6 N \times 6 N}$
$\left[M_{1,1}\right]_{i, j}=\left[M_{2,2}\right]_{i, j}=m \int_{0}^{L} \xi_{i} \xi_{j} d x$
$\left[M_{3,3}\right]_{i, j}=\left[M_{4,4}\right]_{i, j}=I_{s} \int_{0}^{L} \eta_{i} \eta_{j} d x$
$[K]=\left[\begin{array}{cccccc}\mathbf{K}_{1,1} & \mathbf{K}_{1,2} & \mathbf{K}_{1,3} & \mathbf{K}_{1,4} & \mathbf{K}_{1,5} & \mathbf{K}_{1,6} \\ \mathbf{K}_{2,1} & \mathbf{K}_{2,2} & \mathbf{K}_{2,3} & \mathbf{K}_{2,4} & \mathbf{K}_{2,5} & \mathbf{K}_{2,6} \\ \mathbf{K}_{3,1} & \mathbf{K}_{3,2} & \mathbf{K}_{3,3} & \mathbf{K}_{3,4} & \mathbf{K}_{3,5} & \mathbf{0} \\ \mathbf{K}_{4,1} & \mathbf{K}_{4,2} & \mathbf{K}_{4,3} & \mathbf{K}_{4,4} & \mathbf{0} & \mathbf{K}_{4,6} \\ \mathbf{K}_{5,1} & \mathbf{K}_{5,2} & \mathbf{K}_{5,3} & \mathbf{0} & \mathbf{K}_{5,5} & \mathbf{K}_{5,6} \\ \mathbf{K}_{6,1} & \mathbf{K}_{6,2} & \mathbf{0} & \mathbf{K}_{6,4} & \mathbf{K}_{6,5} & \mathbf{K}_{6,6}\end{array}\right]_{6 N \times 6 N}$
$\left[M_{5,5}\right]_{i, j}=\left[M_{6,6}\right]_{i, j}=I_{c} \int_{0}^{L} \eta_{i} \eta_{j} d x$.
$\left[G_{4,3}\right]_{i, j}=2 I_{s} \Omega \int_{0}^{L} \eta_{i} \eta_{j} d x$
$\left[G_{3,4}\right]_{i, j}=-\left[G_{4,3}\right]_{i, j}$,
$\left[G_{6,5}\right]_{i, j}=2 I_{c} \Omega \int_{0}^{L} \eta_{i} \eta_{j} d x$, 


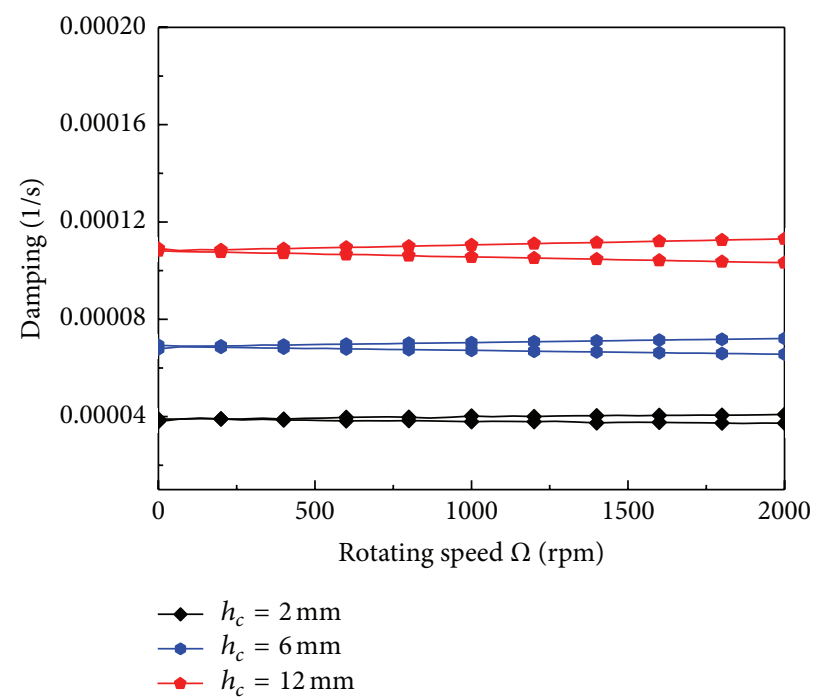

(a) First mode

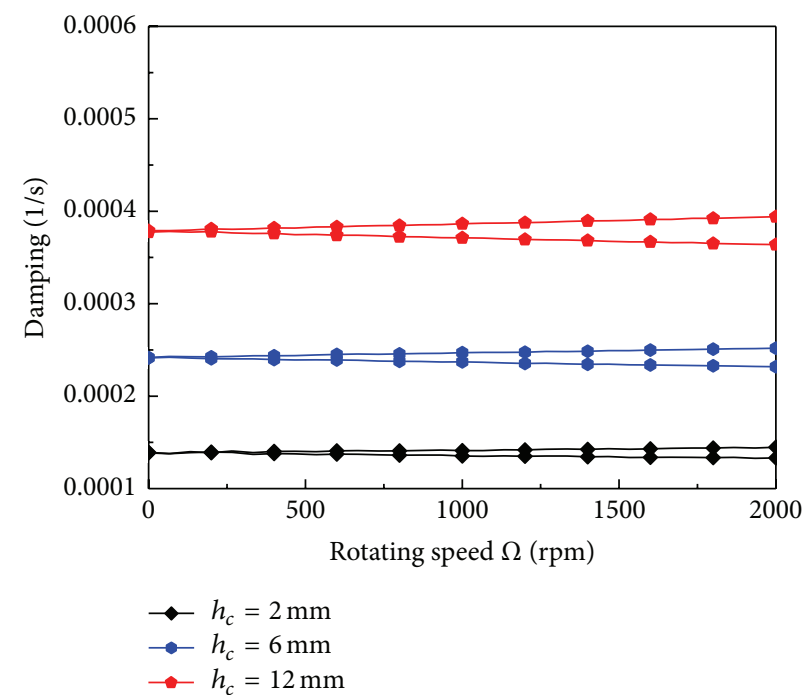

(b) Second mode

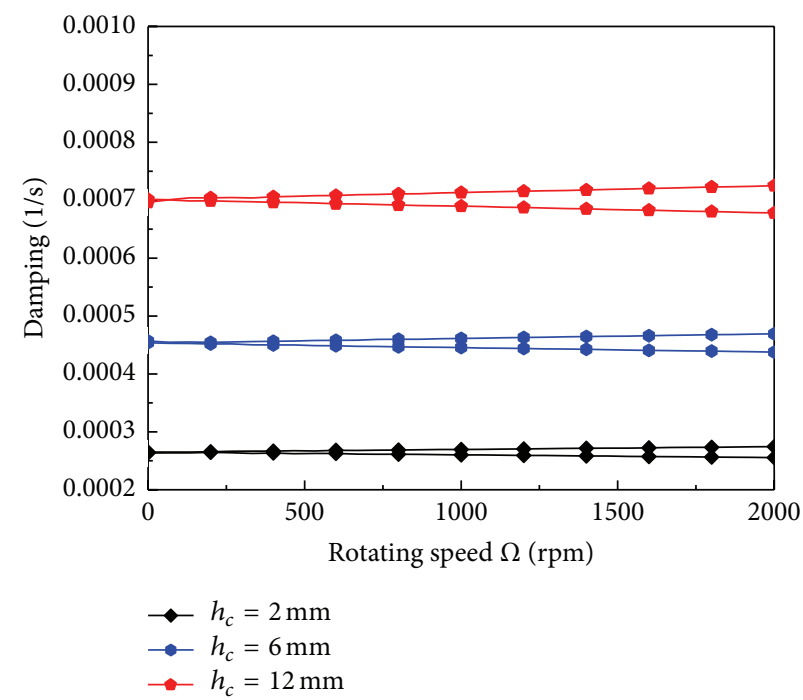

(c) Third mode

FIGURE 7: The first three dampings versus rotating speed for various thicknesses of constraining layer $\left(h_{v}=2 \mathrm{~mm}, \theta=60^{\circ}\right)$.

$$
\begin{array}{ll}
{\left[G_{5,6}\right]_{i, j}=-\left[G_{6,5}\right]_{i, j} \cdot} & {\left[K_{1,6}\right]_{i, j}=B_{21}^{c} \int_{0}^{L} \xi_{i} \eta_{j}^{\prime \prime} d x+k_{5 c} \int_{0}^{L} \xi_{i} \eta_{j}^{\prime} d x,} \\
{\left[K_{1,1}\right]_{i, j}=-k_{1} \int_{0}^{L} \xi_{i} \xi_{j}^{\prime \prime} d x,} & {\left[K_{2,1}\right]_{i, j}=-\left[K_{1,2}\right]_{i, j},} \\
{\left[K_{1,2}\right]_{i, j}=-k_{2} \int_{0}^{L} \xi_{i} \xi_{j}^{\prime \prime} d x,} & {\left[K_{2,2}\right]_{i, j}=\left[K_{1,1}\right]_{i, j},} \\
{\left[K_{1,3}\right]_{i, j}=B_{31}^{s} \int_{0}^{L} \xi_{i} \eta_{j}^{\prime \prime} d x+k_{3} \int_{0}^{L} \xi_{i} \eta_{j}^{\prime} d x,} & {\left[K_{2,3}\right]_{i, j}=B_{21}^{s} \int_{0}^{L} \xi_{i} \eta_{j}^{\prime \prime} d x-k_{5 s} \int_{0}^{L} \xi_{i} \eta_{j}^{\prime} d x,} \\
{\left[K_{1,4}\right]_{i, j}=B_{21}^{s} \int_{0}^{L} \xi_{i} \eta_{j}^{\prime \prime} d x+k_{5 s} \int_{0}^{L} \xi_{i} \eta_{j}^{\prime} d x,} & {\left[K_{2,4}\right]_{i, j}=-B_{31}^{s} \int_{0}^{L} \xi_{i} \eta_{j}^{\prime \prime} d x-k_{3} \int_{0}^{L} \xi_{i} \eta_{j}^{\prime} d x,} \\
{\left[K_{1,5}\right]_{i, j}=B_{31}^{c} \int_{0}^{L} \xi_{i} \eta_{j}^{\prime \prime} d x+k_{4} \int_{0}^{L} \xi_{i} \eta_{j}^{\prime} d x,} & {\left[K_{2,5}\right]_{i, j}=B_{21}^{c} \int_{0}^{L} \xi_{i} \eta_{j}^{\prime \prime} d x-k_{5 c} \int_{0}^{L} \xi_{i} \eta_{j}^{\prime} d x,}
\end{array}
$$



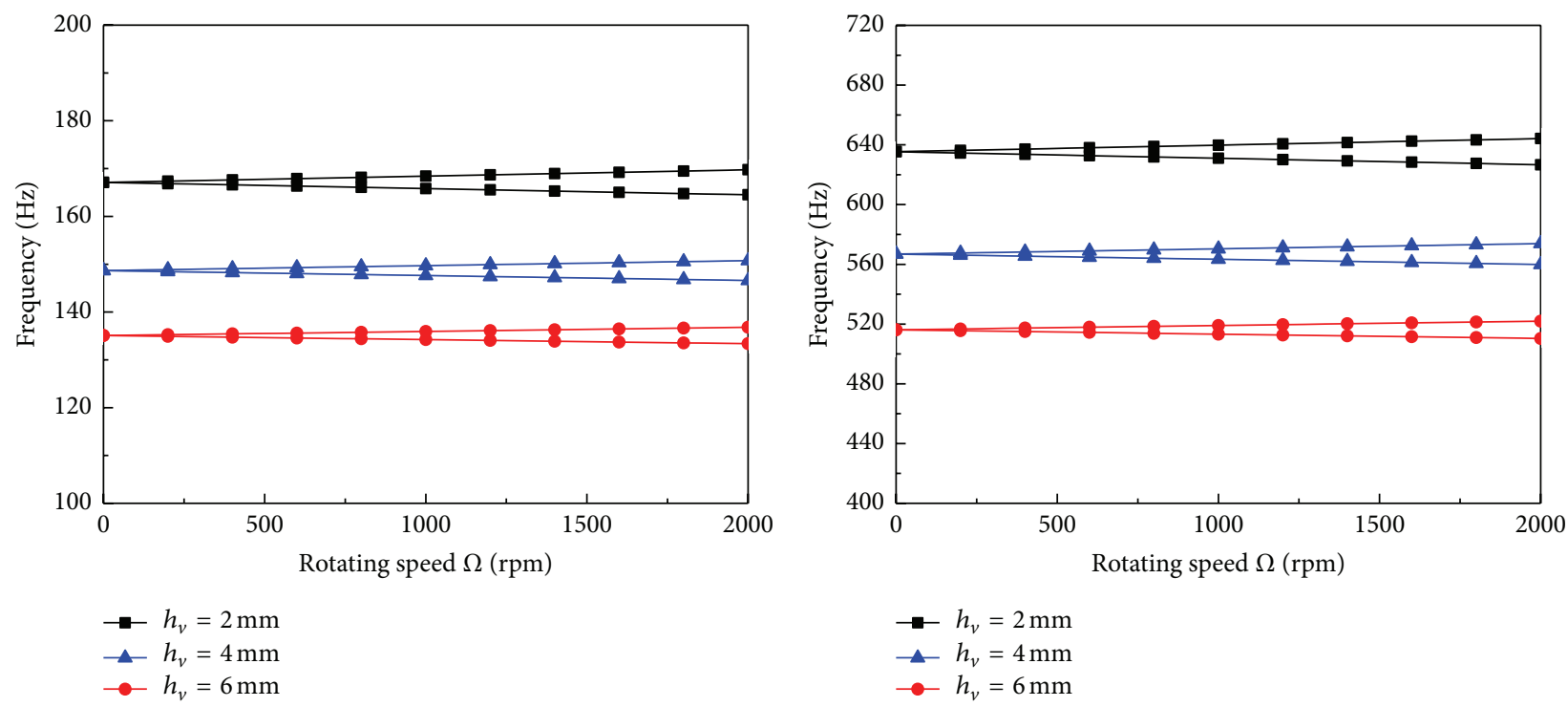

(a) First mode

(b) Second mode

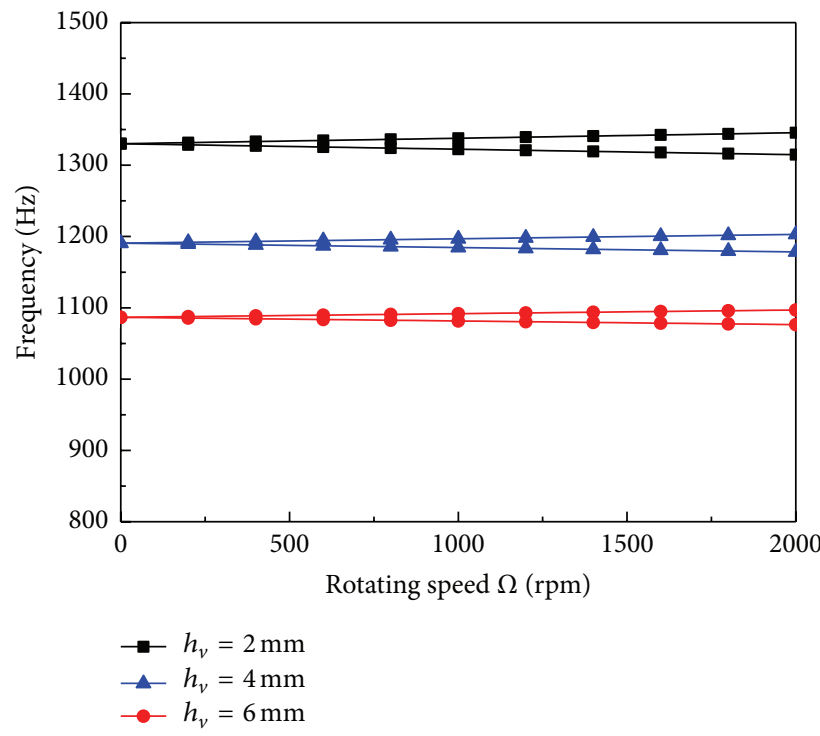

(c) Third mode

FIGURE 8: The first three natural frequencies versus rotating speed for various thicknesses of viscoelastic damping layer $\left(h_{c}=2 \mathrm{~mm}, \theta=60^{\circ}\right)$.

$$
\begin{array}{lr}
{\left[K_{2,6}\right]_{i, j}=-B_{31}^{c} \int_{0}^{L} \xi_{i} \eta_{j}^{\prime \prime} d x-k_{4} \int_{0}^{L} \xi_{i} \eta_{j}^{\prime} d x} & =B_{31}^{s} \int_{0}^{L} \eta_{i} \eta_{j}^{\prime} d x+\kappa B_{13}^{s} \int_{0}^{L} \eta_{i}^{\prime} \eta_{j} d x \\
=-\left[K_{1,5}\right]_{i, j}, & +k_{7} \int_{0}^{L} \eta_{i} \eta_{j} d x+D_{11}^{s} \int_{0}^{L} \eta_{i}^{\prime} \eta_{j}^{\prime} d x, \\
{\left[K_{3,1}\right]_{i, j}=-\kappa B_{13}^{s} \int_{0}^{L} \eta_{i}^{\prime} \xi_{j}^{\prime} d x-k_{6} \int_{0}^{L} \eta_{i} \xi_{j}^{\prime} d x,} & {\left[K_{3,4}\right]_{i, j}} \\
{\left[K_{3,2}\right]_{i, j}=-\kappa B_{12}^{s} \int_{0}^{L} \eta_{i}^{\prime} \xi_{j}^{\prime} d x-k_{5 s} \int_{0}^{L} \eta_{i} \xi_{j}^{\prime} d x,} & =B_{21}^{s} \int_{0}^{L} \eta_{i} \eta_{j}^{\prime} d x-\kappa B_{12}^{s} \int_{0}^{L} \eta_{i}^{\prime} \eta_{j} d x \\
{\left[K_{3,3}\right]_{i, j}} & -k_{5 s} \int_{0}^{L} \eta_{i} \eta_{j} d x,
\end{array}
$$




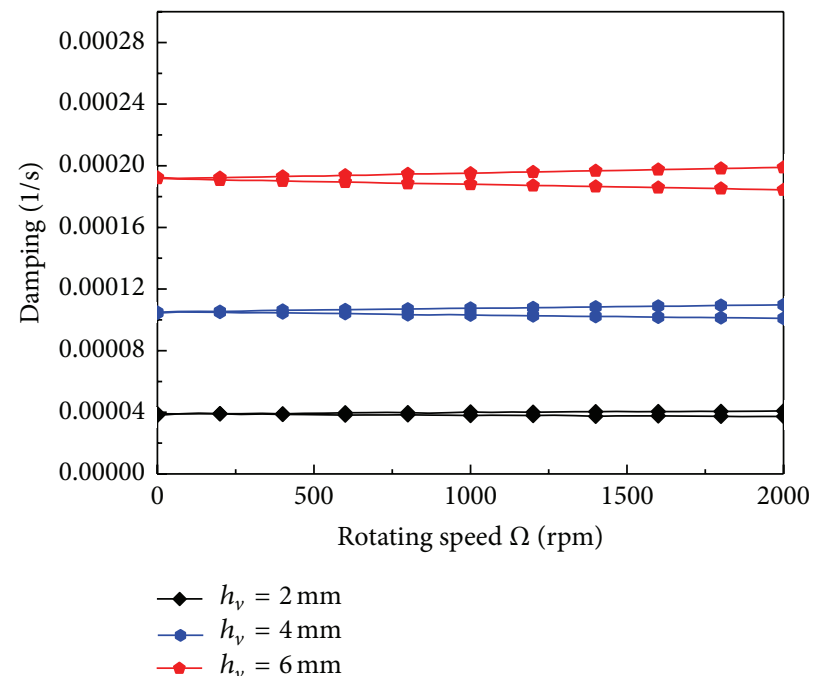

(a) First mode

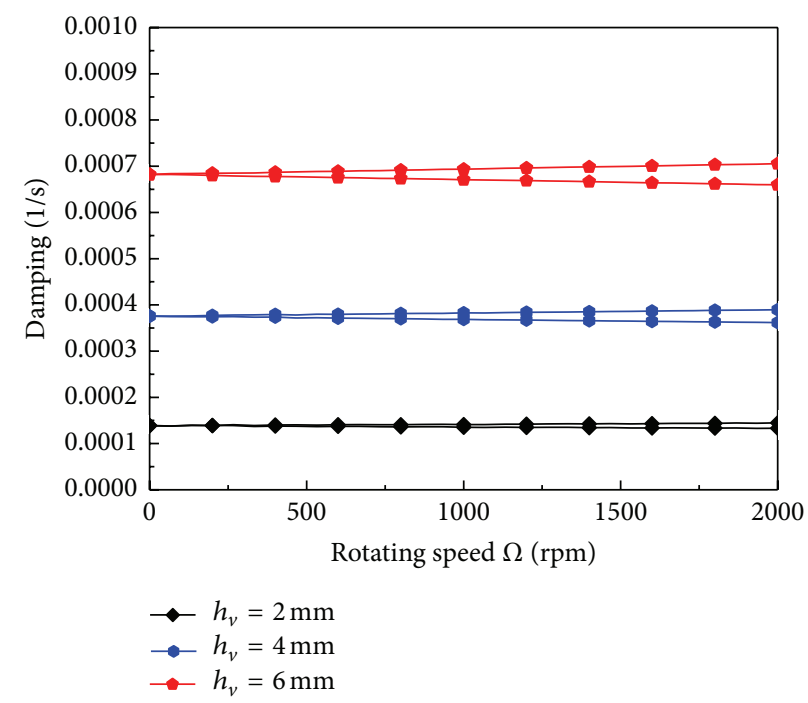

(b) Second mode

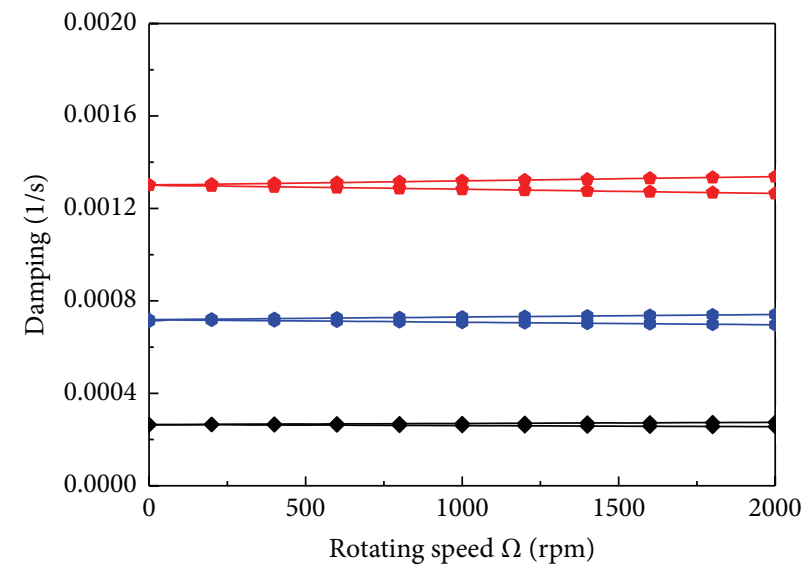

$$
\begin{aligned}
& \multimap h_{v}=2 \mathrm{~mm} \\
& \because h_{v}=4 \mathrm{~mm} \\
& \because h_{v}=6 \mathrm{~mm}
\end{aligned}
$$

(c) Third mode

FIGURE 9: The first three modal dampings versus rotating speed for various thicknesses of viscoelastic damping layer $\left(h_{c}=2 \mathrm{~mm}, \theta=60^{\circ}\right)$.

$$
\begin{aligned}
& {\left[K_{3,5}\right]_{i, j}=-G_{v} A_{v} \frac{\bar{r}_{s c}^{s}}{h_{v}^{2}} \int_{0}^{L} \eta_{i} \eta_{j} d x} \\
& {\left[K_{4,1}\right]_{i, j}=-\kappa B_{12}^{s} \int_{0}^{L} \eta_{i}^{\prime} \xi_{j}^{\prime} d x-k_{5 s} \int_{0}^{L} \eta_{i} \xi_{j}^{\prime} d x} \\
& =\left[K_{3,2}\right]_{i, j}, \\
& {\left[K_{4,2}\right]_{i, j}=\kappa B_{13}^{s} \int_{0}^{L} \eta_{i}^{\prime} \xi_{j}^{\prime} d x+k_{6} \int_{0}^{L} \eta_{i} \xi_{j}^{\prime} d x} \\
& \quad=-\left[K_{3,1}\right]_{i, j}, \\
& {\left[K_{4,3}\right]_{i, j}} \\
& =-B_{21}^{s} \int_{0}^{L} \eta_{i} \eta_{j}^{\prime} d x+\kappa B_{12}^{s} \int_{0}^{L} \eta_{i}^{\prime} \eta_{j} d x
\end{aligned}
$$$$
+k_{5 s} \int_{0}^{L} \eta_{i} \eta_{j} d x=-\left[K_{3,4}\right]_{i, j},
$$$$
\left[K_{4,4}\right]_{i, j}
$$$$
=B_{31}^{s} \int_{0}^{L} \eta_{i} \eta_{j}^{\prime} d x+\kappa B_{13}^{s} \int_{0}^{L} \eta_{i}^{\prime} \eta_{j} d x
$$$$
+k_{7} \int_{0}^{L} \eta_{i} \eta_{j} d x+D_{11}^{s} \int_{0}^{L} \eta_{i}^{\prime} \eta_{j}^{\prime} d x=\left[K_{3,3}\right]_{i, j},
$$$$
\left[K_{4,6}\right]_{i, j}=-G_{v} A_{v} \frac{\bar{r}_{s c}^{s}}{\bar{h}_{v}^{2}} \int_{0}^{L} \eta_{i} \eta_{j} d x=\left[K_{3,5}\right]_{i, j},
$$$$
\left[K_{5,1}\right]_{i, j}=k_{8} \int_{0}^{L} \eta_{i} \xi_{j}^{\prime} d x-\kappa B_{13}^{c} \int_{0}^{L} \eta_{i}^{\prime} \xi_{j}^{\prime} d x,
$$ 


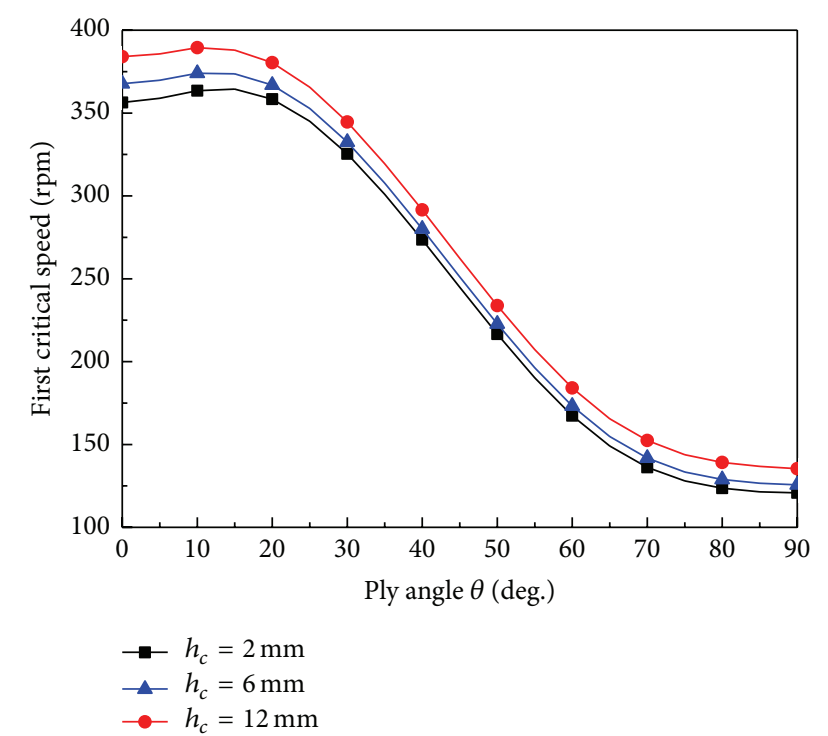

Figure 10: The critical speed versus fiber angle for various thicknesses of constraining layer $\left(h_{v}=2 \mathrm{~mm}\right)$.

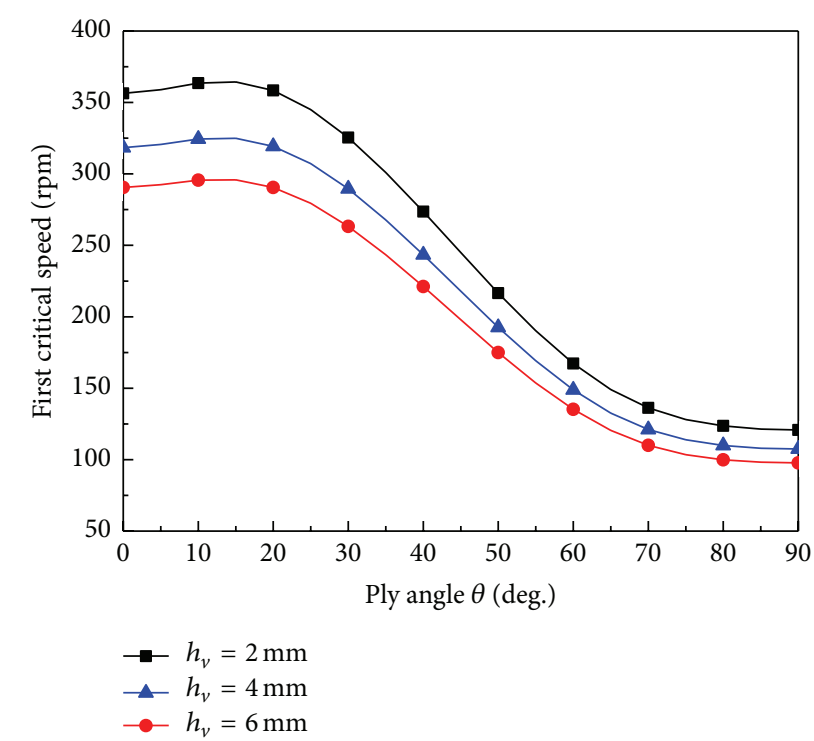

FIGURE 11: The critical speed versus fiber angle for various thicknesses of viscoelastic damping layer $\left(h_{c}=2 \mathrm{~mm}\right)$.

$$
\begin{aligned}
& {\left[K_{5,2}\right]_{i, j}=-k_{5 c} \int_{0}^{L} \eta_{i} \xi_{j}^{\prime} d x-\kappa B_{12}^{c} \int_{0}^{L} \eta_{i}^{\prime} \xi_{j}^{\prime} d x} \\
& {\left[K_{5,3}\right]_{i, j}=-G_{v} A_{v} \frac{\bar{r}_{s c}^{s}}{\bar{h}_{v}^{2}} \int_{0}^{L} \eta_{i} \eta_{j} d x=\left[K_{3,5}\right]_{i, j}} \\
& {\left[K_{5,5}\right]_{i, j}} \\
& =B_{31}^{c} \int_{0}^{L} \eta_{i} \eta_{j}^{\prime} d x+\kappa B_{13}^{c} \int_{0}^{L} \eta_{i}^{\prime} \eta_{j} d x \\
& \quad+k_{9} \int_{0}^{L} \eta_{i} \eta_{j} d x+D_{11}^{c} \int_{0}^{L} \eta_{i}^{\prime} \eta_{j}^{\prime} d x
\end{aligned}
$$

$$
\begin{aligned}
& {\left[K_{5,6}\right]_{i, j}} \\
& =B_{21}^{c} \int_{0}^{L} \eta_{i} \eta_{j}^{\prime} d x-\kappa B_{12}^{c} \int_{0}^{L} \eta_{i}^{\prime} \eta_{j} d x \\
& -k_{5 c} \int_{0}^{L} \eta_{i} \eta_{j} d x \\
& {\left[K_{6,1}\right]_{i, j}=-\kappa B_{12}^{c} \int_{0}^{L} \eta_{i}^{\prime} \xi_{j}^{\prime} d x-k_{5 c} \int_{0}^{L} \eta_{i} \xi_{j}^{\prime} d x} \\
& =\left[K_{5,2}\right]_{i, j}, \\
& {\left[K_{6,2}\right]_{i, j}=-k_{8} \int_{0}^{L} \eta_{i} \xi_{j}^{\prime} d x+\kappa B_{13}^{c} \int_{0}^{L} \eta_{i}^{\prime} \xi_{j}^{\prime} d x} \\
& =-\left[K_{5,1}\right]_{i, j} \text {, } \\
& {\left[K_{6,4}\right]_{i, j}=-G_{v} A_{v} \frac{\bar{r}_{s c}^{s}}{\bar{h}_{v}^{2}} \int_{0}^{L} \eta_{i} \eta_{j} d x=\left[K_{3,5}\right]_{i, j},} \\
& {\left[K_{6,5}\right]_{i, j}} \\
& =-B_{21}^{c} \int_{0}^{L} \eta_{i} \eta_{j}^{\prime} d x+\kappa B_{12}^{c} \int_{0}^{L} \eta_{i}^{\prime} \eta_{j} d x \\
& +k_{5 c} \int_{0}^{L} \eta_{i} \eta_{j} d x=-\left[K_{5,6}\right]_{i, j}, \\
& {\left[K_{6,6}\right]_{i, j}} \\
& =B_{31}^{c} \int_{0}^{L} \eta_{i} \eta_{j}^{\prime} d x+\kappa B_{13}^{c} \int_{0}^{L} \eta_{i}^{\prime} \eta_{j} d x \\
& +k_{9} \int_{0}^{L} \eta_{i} \eta_{j} d x+D_{11}^{c} \int_{0}^{L} \eta_{i}^{\prime} \eta_{j}^{\prime} d x=\left[K_{5,5}\right]_{i, j} . \\
& k_{1}=\kappa\left(A_{22}^{s}+A_{33}^{s}\right)+\kappa\left(A_{22}^{c}+A_{33}^{c}\right)+A_{v} G_{v} \text {, } \\
& k_{2}=\kappa\left(A_{32}^{s}-A_{23}^{s}\right)+\kappa\left(A_{32}^{c}-A_{23}^{c}\right), \\
& k_{3}=\kappa\left(A_{22}^{s}+A_{33}^{s}\right)+A_{v} G_{v} \frac{\bar{r}_{s}}{h_{v}}, \\
& k_{4}=\kappa\left(A_{22}^{c}+A_{33}^{c}\right)-A_{v} G_{v} \frac{\bar{r}_{c}}{h_{v}}, \\
& k_{5 s}=\kappa\left(A_{32}^{s}-A_{23}^{s}\right), \\
& k_{5 c}=\kappa\left(A_{32}^{c}-A_{23}^{c}\right) \text {, } \\
& k_{6}=\kappa\left(A_{22}^{s}+A_{33}^{s}\right)+A_{v} G_{v} \frac{\bar{r}_{s}}{h_{v}}=k_{3} \text {, } \\
& k_{7}=\kappa\left(A_{22}^{s}+A_{33}^{s}\right)+A_{v} G_{v} \frac{\bar{r}_{s s}^{2}}{h_{v}^{2}} . \\
& k_{8}=-\kappa\left(A_{22}^{c}+A_{33}^{c}\right)+A_{v} G_{v} \frac{\bar{r}_{c}}{h_{v}}=-k_{4} \text {, } \\
& k_{9}=\kappa\left(A_{22}^{c}+A_{33}^{c}\right)+A_{v} G_{v} \frac{\bar{r}_{c c}^{2}}{h_{v}^{2}} .
\end{aligned}
$$




\section{B. Matrix Coefficients $\bar{k}_{i j}$ and $\bar{c}_{i j}$}

Matrix coefficients $\bar{k}_{i j}$ and $\bar{c}_{i j}$ in (5) are as follows:

$$
\begin{aligned}
& \bar{k}_{11}^{(k)}=\frac{k_{13}\left(k_{13} k_{22}-k_{12} k_{23}\right)}{\Delta}+\frac{k_{23}\left(k_{11} k_{23}-k_{12} k_{13}\right)}{\Delta} \\
& +k_{33} \\
& \bar{k}_{12}^{(k)}=\frac{k_{13}\left(k_{14} k_{22}-k_{12} k_{24}\right)}{\Delta}+\frac{k_{23}\left(k_{11} k_{24}-k_{12} k_{14}\right)}{\Delta} \\
& +k_{34} \\
& \bar{k}_{13}^{(k)}=0 \text {, } \\
& \bar{k}_{21}^{(k)}=\frac{k_{14}\left(k_{13} k_{22}-k_{12} k_{23}\right)}{\Delta}+\frac{k_{24}\left(k_{11} k_{23}-k_{12} k_{13}\right)}{\Delta} \\
& +k_{34}=\bar{k}_{12}^{(k)} \text {, } \\
& \bar{k}_{22}^{(k)}=\frac{k_{14}\left(k_{14} k_{22}-k_{12} k_{24}\right)}{\Delta}+\frac{k_{24}\left(k_{11} k_{24}-k_{12} k_{14}\right)}{\Delta} \\
& +k_{44} \\
& \bar{k}_{33}^{(k)}=k_{55}-\frac{k_{56}^{2}}{k_{66}}, \\
& \bar{k}_{23}^{(k)}=\bar{k}_{31}^{(k)}=\bar{k}_{32}^{(k)}=0 . \\
& \bar{c}_{11}=\frac{k_{23} k_{21}}{\Delta}-\frac{k_{13} k_{22}}{\Delta} \text {, } \\
& \bar{c}_{12}=\frac{k_{13} k_{12}}{\Delta}-\frac{k_{23} k_{11}}{\Delta}, \\
& \bar{c}_{21}=\frac{k_{42} k_{21}}{\Delta}-\frac{k_{41} k_{22}}{\Delta}, \\
& \bar{c}_{22}=\frac{k_{41} k_{12}}{\Delta}-\frac{k_{42} k_{11}}{\Delta}, \\
& \bar{c}_{31}=\frac{k_{52} k_{21}}{\Delta}-\frac{k_{51} k_{22}}{\Delta}, \\
& \bar{c}_{32}=\frac{k_{51} k_{12}}{\Delta}-\frac{k_{52} k_{11}}{\Delta}, \\
& \Delta=k_{12}^{2}-k_{11} k_{22},
\end{aligned}
$$

where $k_{i j}$ is the off-axis elastic constants of the layer.

\section{Competing Interests}

The authors declare that they have no competing interests.

\section{Acknowledgments}

The research is funded by the National Natural Science Foundation of China (Grant no. 11272190) and Shandong Provincial Natural Science Foundation of China (Grant no. ZR2011EEM031).

\section{References}

[1] H. Zinberg and M. F. Symonds, "The development of an advanced composite tail rotor driveshaft," in Proceedings of the 26th Annual National Forum of the American Helicopter Society, Washington, DC, USA, June 1970.

[2] H. L. M. dos Reis, R. B. Goldman, and P. H. Verstrate, "Thinwalled laminated composite cylindrical tubes; part III-critical speed analysis," Journal of Composites Technology and Research, vol. 9, no. 2, pp. 58-62, 1987.

[3] M.-Y. Chang, J.-K. Chen, and C.-Y. Chang, "A simple spinning laminated composite shaft model," International Journal of Solids and Structures, vol. 41, no. 3-4, pp. 637-662, 2004.

[4] W. Kim, A. Argento, and R. A. Scott, "Free vibration of a rotating tapered composite Timoshenko shaft," Journal of Sound and Vibration, vol. 226, no. 1, pp. 125-147, 1999.

[5] S. P. Singh and K. Gupta, "Composite shaft rotordynamic analysis using a layerwise theory," Journal of Sound and Vibration, vol. 191, no. 5, pp. 739-756, 1996.

[6] H. B. H. Gubran and K. Gupta, "The effect of stacking sequence and coupling mechanisms on the natural frequencies of composite shafts," Journal of Sound and Vibration, vol. 282, no. 1-2, pp. 231-248, 2005.

[7] O. Song, N.-H. Jeong, and L. Librescu, "Implication of conservative and gyroscopic forces on vibration and stability of an elastically tailored rotating shaft modeled as a composite thinwalled beam," Journal of the Acoustical Society of America, vol. 109, no. 3, pp. 972-981, 2001.

[8] R. Sino, T. N. Baranger, E. Chatelet, and G. Jacquet, "Dynamic analysis of a rotating composite shaft," Composites Science and Technology, vol. 68, no. 2, pp. 337-345, 2008.

[9] Y. S. Ren, Q. Y. Dai, and X. Q. Zhang, "Modeling and dynamic analysis of rotating composite shaft," Journal of Vibroengineering, vol. 15, no. 4, pp. 1816-1832, 2013.

[10] D. K. Rao, "Frequency and loss factors of sandwich beams under various boundary conditions," Journal Mechanical Engineering Science, vol. 20, no. 5, pp. 271-282, 1978.

[11] A. Bhimaraddi, "Sandwich beam theory and the analysis of constrained layer damping," Journal of Sound and Vibration, vol. 179, no. 4, pp. 591-602, 1995.

[12] T. Sakiyama, H. Matsuda, and C. Morita, "Free vibration analysis of sandwich beam with elastic or viscoelastic core by applying the discrete green function," Journal of Sound and Vibration, vol. 191, no. 2, pp. 189-206, 1996.

[13] A. Fasana and S. Marchesiello, "Rayleigh-Ritz analysis of sandwich beams," Journal of Sound and Vibration, vol. 241, no. 4, pp. 643-652, 2001.

[14] T. C. Ramesh and N. Ganesan, "Influence of constrained damping layer on the resonant response of orthotropic cylindrical shells," Journal of Sound and Vibration, vol. 185, no. 3, pp. 483500, 1995.

[15] H.-J. Wang and L.-W. Chen, "Finite element dynamic analysis of orthotropic cylindrical shells with a constrained damping layer," Finite Elements in Analysis and Design, vol. 40, no. 7, pp. 737$755,2004$.

[16] A. K. Garg, R. K. Khare, and T. Kant, "Higher-order closedform solutions for free vibration of laminated composite and sandwich shells," Journal of Sandwich Structures and Materials, vol. 8, no. 3, pp. 205-235, 2006.

[17] J. X. Gao and Y. P. Shen, "Vibration and damping analysis of a composite plate with active and passive damping layer," Applied 
Mathematics and Mechanics (English Edition), vol. 20, no. 10, pp. 1075-1086, 1999.

[18] Y.-C. Chen and S.-C. Huang, "An optimal placement of CLD treatment for vibration suppression of plates," International Journal of Mechanical Sciences, vol. 44, no. 8, pp. 1801-1821, 2002.

[19] L. Liu, Z. Zhang, and H. X. Hua, "Dynamic characteristics of rotating cantilever plates with active constrained layer damping treatments," Smart Materials and Structures, vol. 16, no. 5, pp. 1849-1856, 2007.

[20] X.-T. Cao, Z.-Y. Zhang, and H.-X. Hua, "Free vibration of circular cylindrical shell with constrained layer damping," Applied Mathematics and Mechanics (English Edition), vol. 32, no. 4, pp. 495-506, 2011.

[21] G. Jin, C. Yang, Z. Liu, S. Gao, and C. Zhang, "A unified method for the vibration and damping analysis of constrained layer damping cylindrical shells with arbitrary boundary conditions," Composite Structures, vol. 130, pp. 124-142, 2015.

[22] K. L. Napolitano, W. Grippo, J. B. Kosmatka, and C. D. Johnson, "A comparison of two cocured damped composite torsion shafts," Composite Structures, vol. 43, no. 2, pp. 115-125, 1998.

[23] R. Venkatachalam and S. B. Prabu, "Vibration characteristics of orthotropic shaft-disk system with different constrained damping layers: experimental and numerical study," International Journal of Advanced Manufacturing Technology, vol. 65, pp. 601610, 2012.

[24] H. Ghoneim and D. J. Lawrie, "Analysis of the flexural vibration of a composite drive shaft with partial cylindrical constrained layer damping treatment," Journal of Vibration and Control, vol. 12, no. 1, pp. 25-55, 2006. 


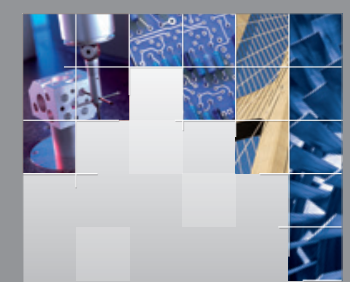

\section{Enfincering}
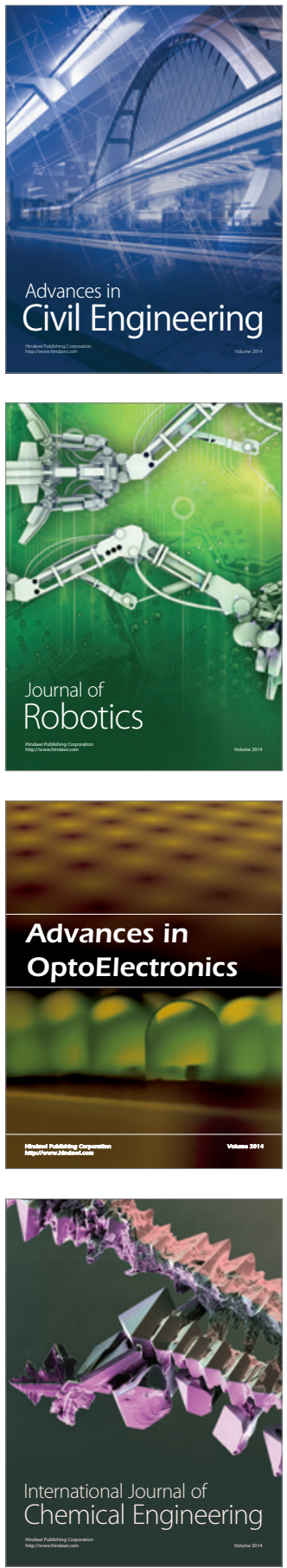

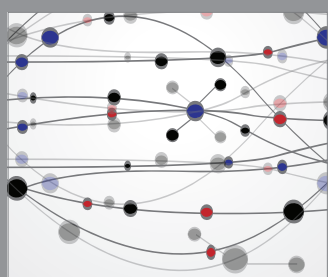

The Scientific World Journal

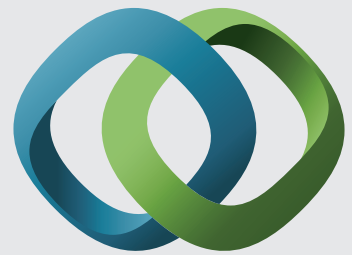

\section{Hindawi}

Submit your manuscripts at

http://www.hindawi.com
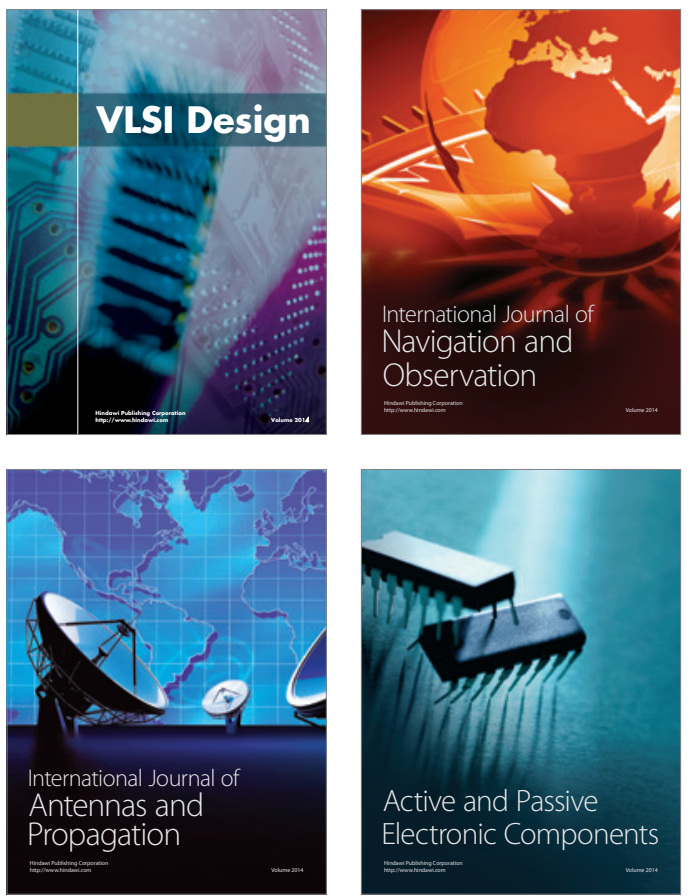
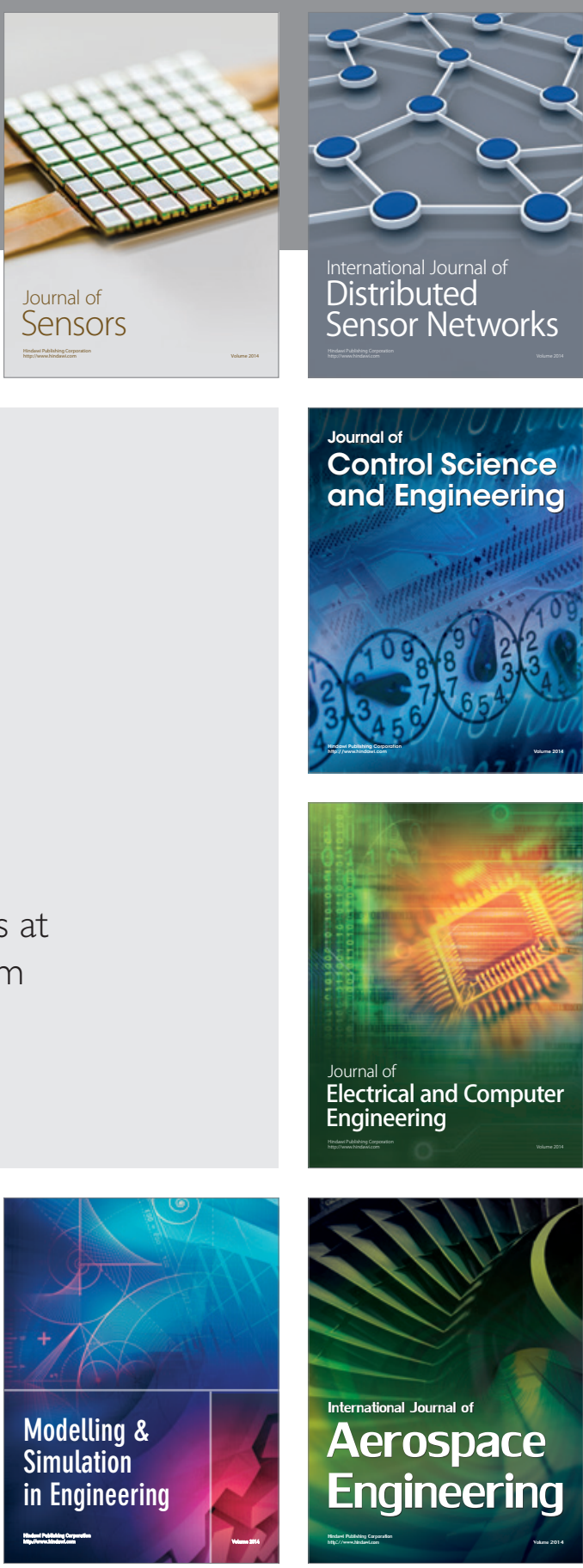

International Journal of

Distributed

Sensor Networks

Journal of

Control Science

and Engineering
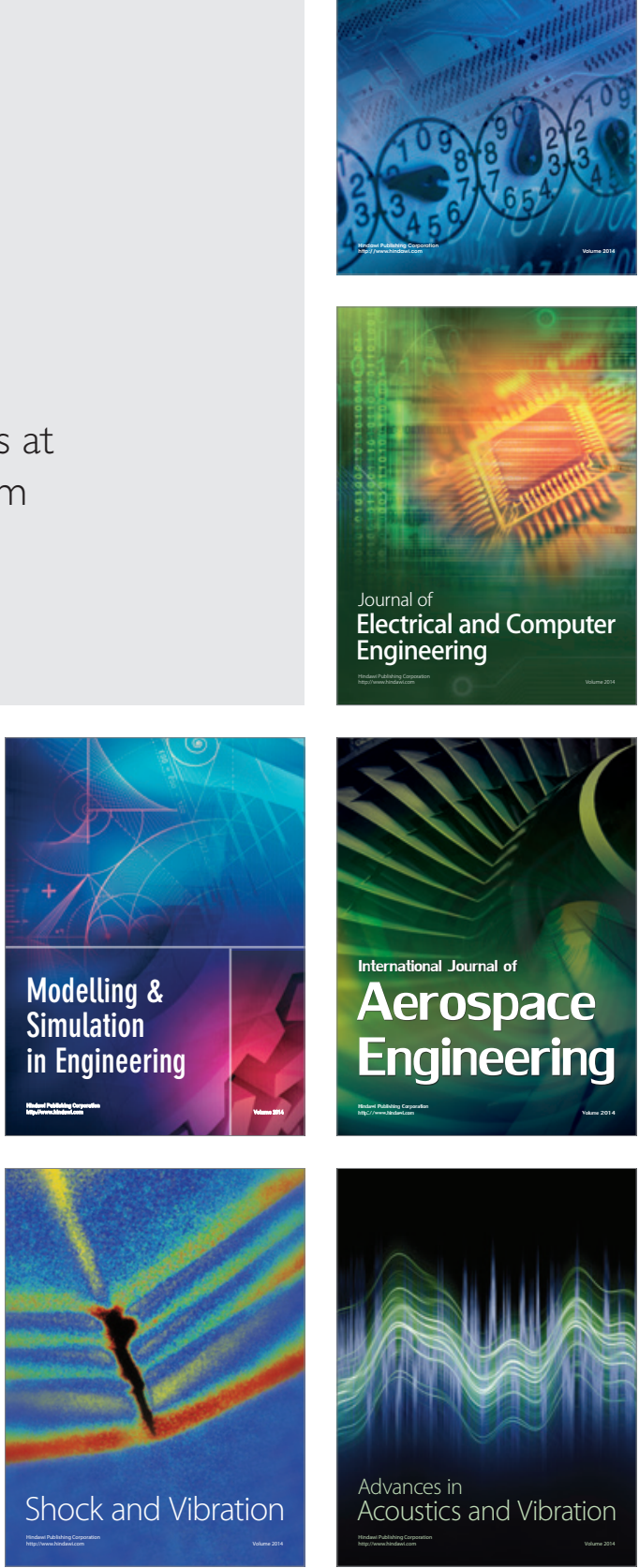